\title{
Convergence of pinching deformations and matings of geometrically finite polynomials
}

\author{
by \\ Peter Haïssinsky (Marseille) and Lei Tan (Cergy-Pontoise)
}

\begin{abstract}
We give a thorough study of Cui's control of distortion technique in the analysis of convergence of simple pinching deformations, and extend his result from geometrically finite rational maps to some subset of geometrically infinite maps. We then combine this with mating techniques for pairs of polynomials to establish existence and continuity results for matings of polynomials with parabolic points. Consequently, if two hyperbolic quadratic polynomials tend to their respective root polynomials radially, and do not belong to conjugate limbs of the Mandelbrot set, then their mating exists and deforms continuously to the mating of the two root polynomials.
\end{abstract}

\section{INTRODUCTION}

Throughout the paper, $d \geq 2$ will denote a fixed integer; the critical set of a polynomial $f$ of degree $d$ is defined as $C(f)=\left\{c: f^{\prime}(c)=0\right\}$, and the postcritical set is

$$
\operatorname{Post}(f)=\bigcup_{c \in C(f)} \overline{\bigcup_{n \geq 1} f^{n}(c)} \text {. }
$$

A rational map $f$ of degree $d$ is said to be geometrically finite (resp. postcritically finite) if its postcritical set has a finite accumulation set (resp. is a finite set). We will say that $f$ is subhyperbolic (resp. hyperbolic) if it is geometrically finite with no parabolic points (resp. with no parabolic points and no critical points on the Julia set). The notion of weakly hyperbolic rational maps generalizes geometrically finite maps to some maps with an infinite postcritical accumulation set. The precise definition will be given later.

Our first task in this paper is to generalize an important result in [4] of Cui from geometrically finite maps to weakly hyperbolic maps:

2000 Mathematics Subject Classification: Primary 37F10; Secondary 37F30.

Research of the second author partially supported by British EPSRC Grant GR/L60999. 
THEOREM A. Let $f_{0}$ be a weakly hyperbolic rational map with attracting points. Let $\left(\gamma_{i}\right)$ be a collection of $f_{0}$-periodic cycles of star-like graphs linking a repelling central vertex to attracting vertices. Then there is a continuous path $\left(f_{t}\right)_{t \in[0,1)}$ of qc-deformations of $f_{0}$, called SIMPLE PINCHING DEFORMATIONS, converging uniformly to a limit rational map $f_{1}$, shrinking progressively each graph $\gamma_{i}$ to a parabolic point for $f_{1}$. All the connected components of the preimages of these graphs are also shrunk, and these are the only changes in the limit.

Detailed definitions and a more precise statement of Theorem A will be given in Section 2.1.

This result is actually more subtle than it appears to be. Although it is fairly easy to imagine the existence of a parabolic map with the right combinatorics, there might be many such maps, especially in the presence of several critical points in the basin. It is then quite surprising that the pinching path accumulates to a single parabolic map.

This theorem, combined with other techniques, creates many interesting existence and continuity results. Cui's original work has used this to obtain a topological characterization of geometrically finite rational maps, which generalizes Thurston's theorem on postcritically finite rational maps. See [4] and [27] for further details.

Here we combine it with the technique of matings of polynomials, and thus answer affirmatively a question raised by Milnor: Can the mating of two hyperbolic quadratic polynomials be deformed continuously to a mating of two parabolic polynomials?

A marked mating is, roughly speaking, a 4-tuple $(f, g, q, R)$ with $f$ and $g$ two degree $d$ monic polynomials having connected and locally connected filled Julia set $K_{f}$ and $K_{g}$, with $R: \overline{\mathbb{C}} \rightarrow \overline{\mathbb{C}}$ a rational map, and with $q$ a homeomorphism from $K_{f} \sqcup K_{g} /\left(\gamma_{f}(t) \sim \gamma_{g}(-t)\right)$ to $\overline{\mathbb{C}}$ conjugating the quotient dynamics induced by $f$ and $g$ to the dynamics of $R$, where $\gamma_{f}$ : $\mathbb{R} / \mathbb{Z} \rightarrow \partial K_{f}$ (resp. $\gamma_{g}$ ) is the Carathéodory semiconjugacy. We establish:

Theorem B. Let $\left(f_{0}, g_{0}, q_{0}, R_{0}\right)$ be a marked mating of geometrically finite polynomials with connected Julia sets and attracting points. Given a simple pinching path $\left(f_{t}\right)_{t \in[0,1)}$ of $f_{0}$ and a simple pinching path $\left(g_{t}\right)_{t \in[0,1)}$ of $g_{0}$, there is a simple pinching path $\left(R_{t}\right)_{t \in[0,1)}$ of $R_{0}$ together with a continuous path of maps $q_{t}$ such that

1. $\left(f_{t}, g_{t}, q_{t}, R_{t}\right)$ is a geometric mating for every $t \in[0,1)$ and depends continuously on $t$.

2. The three pinching paths $\left(f_{t}\right),\left(g_{t}\right)$ and $\left(R_{t}\right)$ converge to $f_{1}, g_{1}$ and $R_{1}$ respectively.

3. The maps $q_{t}$ converge uniformly to a map $q_{1}$.

4. The quadruple $\left(f_{1}, g_{1}, q_{1}, R_{1}\right)$ is a marked mating with parabolic points. 
Schematically, the following diagram is well defined and commutative:

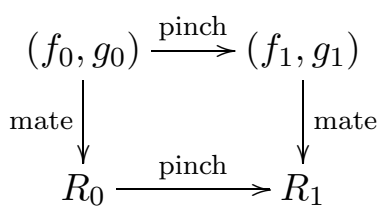

We will give a more precise definition of marked matings in Section 3 , especially we will make all $q_{t}$ defined on the same space in order to talk about their continuity in $t$.

This theorem is to be compared with the examples constructed by Epstein [8] showing that there are paths of marked matings $\left(f_{t}, g_{t}, q_{t}, R_{t}\right)$ where $f_{t}$ and $g_{t}$ converge but $R_{t}$ accumulates to a large set of limit parabolic maps. In these examples the multipliers of the corresponding attracting cycles tend to 1 along a horocycle, whereas pinching produces multipliers that converge to 1 radially, in the sense of McMullen (see [17]).

Applying these results to the quadratic polynomials $f_{c}: z \mapsto z^{2}+c$, $c \in \mathbb{C}$, we get:

Corollary C. Two geometrically finite maps $f_{c}$ and $f_{c^{\prime}}$ are matable if and only if $\bar{c}$ and $c^{\prime}$ do not belong to the same limb of the Mandelbrot set.

Combining Theorem B with techniques of perturbations of parabolics, we can actually obtain a more general parabolic $\leftrightarrow$ attracting-closing-lemma which we state as Theorems D and E: to each geometrically finite polynomial with connected Julia set can be associated a postcritically finite polynomial $\mathcal{T}(f)$ of the same degree with a homeomorphic Julia set and with conjugate dynamics on their Julia sets (see details below).

TheOREM D. Two geometrically finite polynomials $f$ and $g$ with connected Julia sets and parabolic points are matable if and only if $\mathcal{T}(f)$ and $\mathcal{T}(g)$ are matable.

The following theorem gives more information:

Theorem E. Let $f$ and $g$ be two matable geometrically finite polynomials with connected Julia sets and parabolic points. Then there exist subhyperbolic perturbations $\left(f_{t}\right)_{t \in[0,1)}$ and $\left(g_{t}\right)_{t \in[0,1)}$ which converge to $f$ and $g$ respectively as $t$ tends to 1 such that $J_{f_{t}} \approx J_{f}, J_{g_{t}} \approx J_{g}$, and their matings exist and converge to a mating of $f$ and $g$.

In $\S 2$ we restate and prove Theorem A. For this we provide a thorough study of Cui's control of distortion technique. This technique is an important innovation to complex dynamics and will surely find wide applicability. The global structure of our proof is somewhat different from Cui's original one, and our definition of pinching is somewhat more general. We also use a different argument reducing the study around parabolic points to the anal- 
ysis of a simple model system. In $§ 3$ we recall basic definitions and results about matings of polynomials and then apply Theorem A to study matings of geometrically finite polynomials. The paper also contains two appendices which may be of independent interest.

Background on complex dynamics can be found in $[3,19]$ and on quasiconformal maps in [1]. We also assume that the reader is familiar with basic quasiconformal surgeries as those explained in [3].

Acknowledgements. This work grew out from a question raised by John Milnor to Tan Lei about the radial continuity of matings at root quadratic polynomials. We are grateful to him for having induced our project. We would also like to thank Cui Guizhen for enlightening explanations on his work, and Kevin Pilgrim and the anonymous referee for their valuable comments which have enabled us to improve the exposition.

\section{PINCHING DEFORMATION OF RATIONAL MAPS-THEOREM A}

2.1. Definition and the pinching theorem. Let $f$ be a rational map. Denote by $J_{f}$ the Julia set and by $\mathcal{F}$ the Fatou set. A simple pinching combinatorics is a finite collection of $\gamma_{i}$ satisfying:

- Each $\gamma_{i}$ is a repelling star-like closed graph in the following sense:

- the central vertex $\beta_{i}$ is a repelling periodic point, and is not in the $\omega$-limit of recurrent critical points;

- every edge $\kappa$ links $\beta_{i}$ to an attracting periodic point $\alpha$, and there are no other edges between these two vertices; further, for $q$ the period of $\alpha$, we have $f^{q}(\kappa)=\kappa$ and $\left(f^{q}, B^{\prime}\right)$ is conformally conjugate to the translation by 1 on a horizontal strip (where $B^{\prime}$ is a neighborhood of $\kappa \backslash\left\{\alpha, \beta_{i}\right\}$ ); for simplicity, we also require that $\kappa$ intersects the boundary of any linearizable disk around $\alpha$ at only one point (examples of such $\left(\kappa, B^{\prime}\right)$ are suitable straight lines and strips in the log-linearizing coordinates of $\alpha$ );

$-\gamma_{i} \backslash\left\{\beta_{i}\right\} \subset \mathcal{F}$ and is disjoint from the orbits of the critical points.

- The $\gamma_{i}$ 's are mutually disjoint.

- $f: \gamma_{i} \rightarrow \gamma_{j}$ is a homeomorphism.

(The simplest example of a simple pinching combinatorics is the segment $[0,1 / 2]$ for $f(z)=z^{2}+z / 2$.)

This collection of $\gamma_{i}$ can be decomposed into $n_{c}$ cycles. Set $\widehat{R}=\bigcup_{i} \gamma_{i}$, let $\left\{\widehat{R}_{i}\right\}_{1 \leq i \leq n_{c}}$ be the set of cycles and $\mathcal{R}=\bigcup_{n} f^{-n}(\widehat{R})$. The definition above guarantees that each $\mathcal{R}$-component $R$ is again star-like, with a unique Julia point $\beta(R)$ (the word "simple" refers precisely to this fact). We make a (by 
no means canonical) choice of three distinct points $a, b, c$ such that no two belong to the same component of $\mathcal{R}$.

Definition of weak hyperbolicity. We say that $f$ is weakly hyperbolic if there are constants $r>0$ and $\delta<\infty$ such that, for all $z \in J_{f} \backslash$ \{preparabolic points\}, there is a subsequence of iterates $\left(f^{n_{k}}\right)_{k}$ such that

$$
\operatorname{deg}\left(f^{n_{k}}: W_{k}(z) \rightarrow D\left(f^{n_{k}}(z), r\right)\right) \leq \delta,
$$

where $W_{k}(z)$ is the connected component of $f^{-n_{k}}\left(D\left(f^{n_{k}}(z), r\right)\right)$ containing $z$. A simple uniform continuity argument implies that this definition is invariant under topological conjugacies.

Let us remark that if $f$ is hyperbolic, then $f$ is also weakly hyperbolic, and we can choose $\delta=1$ and $r=\frac{1}{2} \operatorname{dist}\left(J_{f}, \operatorname{Post}(f)\right)$. It can be shown that geometrically finite rational maps are weakly hyperbolic.

The following is a restatement of Theorem A, in a more precise form:

THEOREM 2.1. Assume that $f$ is a weakly hyperbolic rational map. Let $\left(\gamma_{i}\right)$ be a simple pinching combinatorics for $f$. Then there is a convergent continuous path $f_{t}$ of $K_{t}$-qc-deformations of $f\left(\right.$ with $\left.K_{t} \rightarrow \infty\right)$, shrinking progressively each $\gamma_{i}$ to a parabolic point, and making no other changes.

More precisely, there is a continuous path of complex structures $\sigma_{t}$, with $\sigma_{0}$ the standard complex structure, such that for $h_{t}$ the integrating map of $\sigma_{t}$ fixing $a, b, c$, and for $f_{t}=h_{t} \circ f \circ h_{t}^{-1}$, we have

(I) $h_{t} \rightrightarrows H$.

(II) The nontrivial fibers of $H$ coincide with the $\mathcal{R}$-components.

(III) $f_{t} \rightrightarrows F$.

(IV) $F \circ H=H \circ f$ and $\left.H\right|_{J_{f}}: J_{f} \rightarrow J_{F}$ is a homeomorphism.

Let us note that $h_{0}$ is the identity since it integrates the standard complex structure and it fixes three points.

Throughout the paper the sign $\rightrightarrows$ will mean uniform convergence.

This theorem is a generalization of a work of Cui [4] who proved the same result for geometrically finite maps. Our proof uses a fundamental idea of Cui (see the Key Lemma below), and follows essentially the same lines as his original proof, but with a different presentation, and a somewhat more general definition of the pinching deformation.

Definition of $\sigma_{t}$. We will use different colors to design regions with special properties. Roughly speaking, the red set is the set to be pinched. The yellow set surrounds the red one and contains the support of $\sigma_{t}$. The green (resp. blue) set is a neighborhood of the yellow acting as a protecting neighborhood, in the sense that on green $\backslash$ yellow and on blue $\backslash$ green we are sure that there are no deformations. Note that some regions have several colorings. 
Although these notions look very complicated, pinching is fundamentally a very simple operation to create parabolic points. But to guarantee the convergence, and that the limit has no other accidental changes in the dynamics, we need more precise information about where the deformation occurs and where not. The yellow set is assigned to locate the support of deformations. But we do not know how big its complement is, especially when we get close to a Julia point. The two consecutive protecting neighborhoods, green and blue, are assigned to guarantee some definite, undeformed space around the yellow set. In most cases the green neighborhood is enough. One appreciates best its importance in the Key Lemma below. The second neighborhood, blue, is however fundamental in the analysis around parabolic points, and in the proof that the limit dynamics is again weakly hyperbolic. For instance it will enable us to get bounds in the green set of the distortion of $p$-valent maps.

Here is a detailed description. We will first define appropriate quasiconformal deformations on some model strips and then implement them into the dynamical plane. In the model, we have cut the star along the red curve so it is doubled (cf. $\S 1$ in [12] for a similar construction).

Our model spaces will be closed horizontal strips on upper or lower halfplanes.

Choose a collection of numbers $0<L_{b}<L_{g}<L_{y}<L_{r}$ (the indices $b, g, y, r$ are colors, blue, green, yellow and red respectively), and then an increasing $C^{1}$-function $\tau:\left[0,1\left[\rightarrow\left[L_{r}, \infty\left[\right.\right.\right.\right.$. Let $M \subset \mathbb{R}^{2}$ be the closed subset bounded by

$$
\left([0,1] \times\left\{L_{b}\right\}\right) \cup\left(\{0\} \times\left[L_{b}, L_{r}\right]\right) \cup\left(\{1\} \times\left[L_{b}, \infty[) \cup\{(t, \tau(t)): t \in[0,1[\} .\right.\right.
$$

Choose $v_{t}(y)$ so that $v_{t}(y)=y$ for $L_{b} \leq y \leq L_{y}$ and that $(t, y) \mapsto\left(t, v_{t}(y)\right)$ is a $C^{1}$-diffeomorphism from $[0,1] \times\left[L_{b}, L_{r}\right] \backslash\left\{\left(1, L_{r}\right)\right\}$ onto $M$.

We also make the following technical assumption: For any $L^{\prime}<L_{r}$, there is $\left.t\left(L^{\prime}\right) \in\right] 0,1\left[\right.$ with $t\left(L^{\prime}\right) \rightarrow 1$ as $L^{\prime} \rightarrow L_{r}$ such that for any $(s, y) \in$ ]$\left.t\left(L^{\prime}\right), 1\right] \times\left[L_{b}, L^{\prime}\right]$, we have $v_{s}(y)=v_{t\left(L^{\prime}\right)}(y)$. This assumption will be used only once in the proof of Lemma 2.8 .

Now on the straight strip $\left\{x+i y: L_{b} \leq y \leq L_{r}\right\}$, and for every $t \in[0,1]$, set

$$
\widetilde{P}_{t}(x+i y)=x+i \cdot v_{t}(y) .
$$

This map has the following properties (cf. Fig. 1):

1. It commutes with translation by 1 (and by any other real number).

2. It is the identity on the substrip $\left\{L_{b} \leq y \leq L_{y}\right\}$.

3. The coefficient of the Beltrami form

$$
\left.\frac{\partial \widetilde{P}_{t} / \partial \bar{z}}{\partial \widetilde{P}_{t} / \partial z}\right|_{x+i y}=\frac{1-\frac{\partial}{\partial y} v_{t}(y)}{1+\frac{\partial}{\partial y} v_{t}(y)}
$$




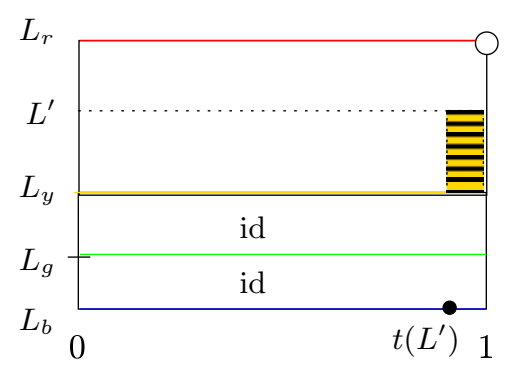

Fig. 1. The diffeomorphism $(t, y) \mapsto\left(t, v_{t}(y)\right)$

is continuous on $(t, x+i y) \in[0,1] \times\left\{L_{b} \leq y \leq L_{r}\right\}$, its norm is locally uniformly bounded away from 1 if $(t, y) \neq\left(1, L_{r}\right)$ and tends to 1 as $(t, y) \rightarrow\left(1, L_{r}\right)$.

4. The map $P_{t}(z)=-1 / \widetilde{P}_{t}(-1 / z)$ is continuous in $(t, z)$. For $t<1, P_{t}$ is injective.

Let us now define appropriate domains in the dynamical space which will support our deformation. Choose one edge in each edge orbit of $\widehat{R}$. Let $\kappa$ be one such edge, of period $q$, and let $B^{\prime}$ be its invariant strip neighborhood $\left(\right.$ for $f^{q}$ ). Let $B^{\prime l}$ denote the component of $B^{\prime} \backslash \kappa$ with $\kappa$ as the left boundary (following the direction of the dynamics) and let $B^{\prime r}$ be the other component.

By assumption, there are $0<L_{b}<L_{r}$ such that $\left(f^{q}, B^{\prime l}\right)$ is conjugate via a conformal map $\psi$ to $\left(z \mapsto z+1,\left\{L_{b}<\operatorname{Im} z<L_{r}\right\}\right)$ and $\psi(\kappa)=\left\{\operatorname{Im} z=L_{r}\right\}$. Define as above a deformation $\widetilde{P}_{t}$ on the model strip $\left\{L_{b}<\operatorname{Im} z<L_{r}\right\}$, and for $t \in\left[0,1\left[\right.\right.$, set $\sigma_{t}^{\prime}=\left(\widetilde{P}_{t} \circ \psi\right)^{*}\left(\sigma_{0}\right)$ to be the pulled back complex structure on $B^{\prime l}$. 
For the other half neighborhood $B^{\prime r}$ of $\kappa$, use a complex conjugate model dynamics $\left(z \mapsto z+1,\left\{-L_{r}^{\prime}<\operatorname{Im} z<-L_{b}^{\prime}<0\right\}\right)$ and complex conjugate deformations (so that $\kappa$ always corresponds to the red boundary line). Note that the left and right model strips are not necessarily symmetric and we do not require symmetric deformations. We will use nevertheless the same letters for convenience.

We may then define $Y(\kappa)$ (resp. $G^{\prime}(\kappa), B^{\prime}(\kappa)$ ), the yellow (resp. green, blue) strip neighborhood, to be $\{\alpha\} \cup \psi\left(\left\{|\operatorname{Im} z| \geq L_{y}\right\}\right)$ (resp. $\psi(\{|\operatorname{Im} z|$ $\left.\left.\left.>L_{g}\right\}\right), \psi\left(\left\{|\operatorname{Im} z|>L_{b}\right\}\right)=B^{\prime}\right)$. The green neighborhood $G(\kappa)$ will be of the form $G^{\prime}(\kappa) \cup \Delta_{\alpha}^{\prime}$ with $\Delta_{\alpha}^{\prime}$ a suitable neighborhood of the attracting end $\alpha$, and the blue neighborhood $B(\kappa)$ will be of the form $B^{\prime}(\kappa) \cup \Delta_{\alpha} \cup \Delta_{\beta}$, where $\Delta_{\alpha}$ and $\Delta_{\beta}$ are suitable neighborhoods of the attracting end $\alpha$ and of the center $\beta$, such that $\overline{G(\kappa)} \subset B(\kappa)$.

Set $\sigma_{t}=\bigcup_{n}\left(f^{n}\right)^{*}\left(\sigma_{t}^{\prime}\right)$. It is an $f$-invariant complex structure, and is conformal outside the grand orbit $\mathcal{Y}$ of $\bigcup_{\kappa} Y(\kappa)$.

Definition of a simple pinching deformation. A simple pinching deformation supported by the simple pinching combinatorics $\widehat{R}$ is given by the family of $f$-invariant complex structures $\sigma_{t}$. We say that the deformation is convergent if the conclusions of Theorem 2.1 are satisfied for some homeomorphisms $h_{t}$ integrating $\sigma_{t}$ and maps $f_{t}=h_{t} \circ f \circ h_{t}^{-1}$.

Scheme of the proof. We will prove first that $\left(h_{t}\right)$ is equicontinuous at every point $z_{0}$ (which would imply uniform equicontinuity). To do this, we will distinguish essentially three cases: $z_{0} \notin J_{f} \cup \mathcal{R}, z_{0} \in J_{f} \backslash(\mathcal{R} \cup$ \{preparabolics\}), and $z_{0} \in \mathcal{R} \cup\{$ preparabolics\}. While the equicontinuity in the first case is more or less automatic, in the last two cases it depends on estimates under deformation of the moduli of many annuli, that we will control thanks to a clever argument of Cui.

Once we know that $\left(h_{t}\right)$ is an equicontinuous family, we will study the fiber structure of any limit map and show that it satisfies the conclusions of the theorem. This will enable us to prove that $\left(f_{t}\right)$ is equicontinuous and that any limit map is again weakly hyperbolic. This will in turn imply that two limits of $\left(f_{t}\right)$ have to be topologically conjugate, with a conjugacy that is conformal off the Julia sets. We can then use a rigidity result of Haïssinsky to conclude that this conjugacy is in fact the identity. This shows the convergence of the pinching deformation.

Notation. In this last introductory section, we define families of sets associated to $\mathcal{R}$ (see Fig. 2). We use the assumption that the repelling ends are disjoint from the $\omega$-limit set of recurrent critical points to show that these sets have bounded geometry (Lemma 2.2).

For each cycle $\widehat{R}_{i}, 1 \leq i \leq n_{c}$, of $\widehat{R}$, we choose a connected component $\gamma_{i}$ with center $\beta_{i}$. We also pick an attracting point $\alpha_{i, j}$ for each attracting 
$Y=$ closed shaded strip $\backslash\{\beta\}$ (contains the support of $\sigma_{t}$ )

$Y^{*}=Y \cup\{\beta\}$

$G^{\prime}=$ first protecting strip of $Y \backslash\{\alpha\}$

$G=G^{\prime} \cup \Delta_{\alpha}^{\prime}$ (open and bounded by the dotted curve)

$G^{*}=G \cup\{\beta\}$ (neither open nor closed)

$B^{\prime}=$ second protecting strip of $Y \backslash\{\alpha\}$

$B=B^{\prime} \cup \Delta_{\alpha} \cup \Delta_{\beta}$ (bounded by the dashed curve)

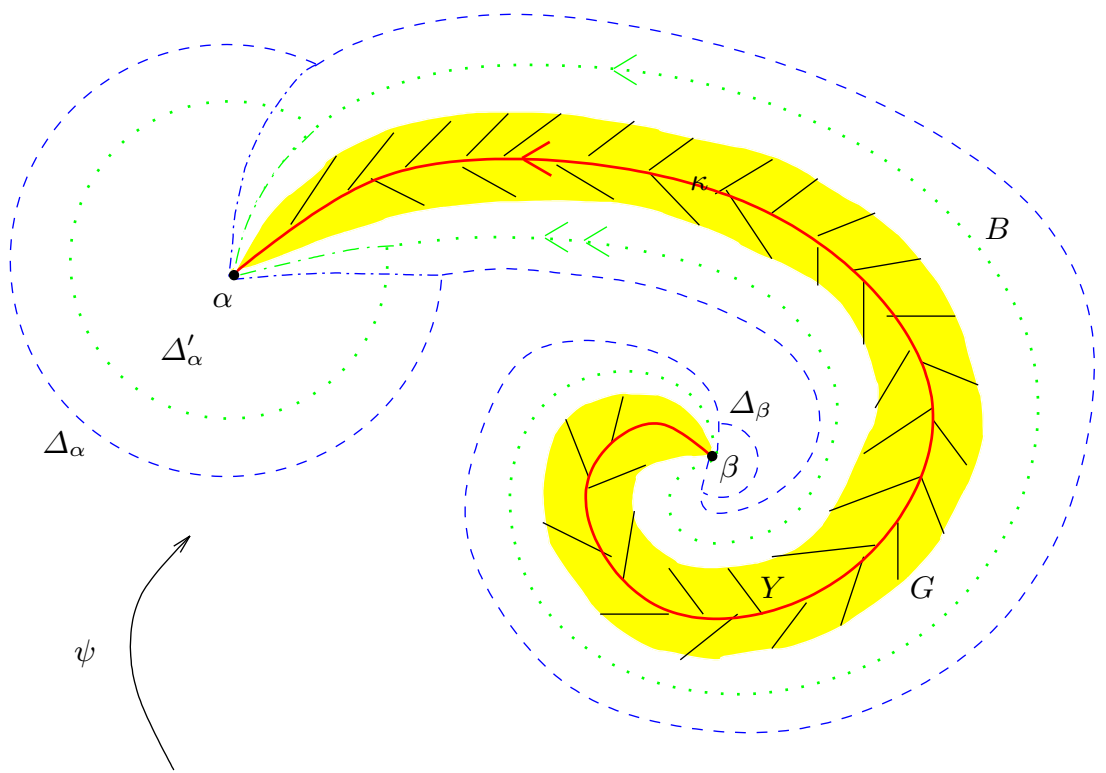

A left model strip

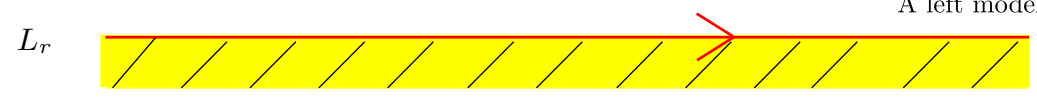

$L_{g}$

$L_{b}$

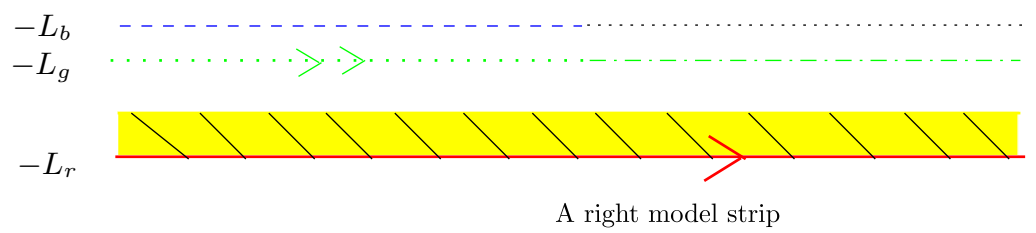

Fig. 2. Deformation strip and protecting neighborhoods

cycle attached to $\beta_{i}$. If $\beta_{i}$ is $k_{i}$-periodic then each $\alpha_{i, j}$ is $k_{i} c_{i}$-periodic for some $c_{i} \geq 1$. We will first define suitable linearizable disks for each of these points. Let us recall that when $f$ is a holomorphic germ which fixes a point $\alpha$ such that $\left|f^{\prime}(\alpha)\right| \notin\{0,1\}$, then there is a linearizing coordinate, that is, a univalent map $\xi$ defined from a neighborhood of $\alpha$ onto a neighborhood $N$ 
of 0 such that $\xi \circ f(z)=f^{\prime}(\alpha) \cdot \xi(z)$. The preimage of any disk centered at the origin contained in $N$ is by definition a linearizable disk for $\alpha$.

Let us start with an attracting cycle. We let $\mathcal{C}=\left\{\alpha_{i, j}, \ldots, f^{c_{i} k_{i}}\left(\alpha_{i, j}\right)\right\}$ and $\mathcal{C}^{\prime}=f^{-1}(\mathcal{C})$. For each $\alpha \in \mathcal{C}^{\prime}$, there is a minimal iterate $\ell=\ell(\alpha) \geq 0$ such that $f^{\ell}(\alpha)=\alpha_{i, j}$. There is also a neighborhood $N=N(\alpha)$ of $\alpha$ such that $\left.f^{\ell}\right|_{N}$ is univalent and $f^{\ell}(N)$ is contained in a linearizable disk for $\alpha_{i, j}$. For $\alpha \in \mathcal{C}^{\prime} \backslash \mathcal{C}$, we may also assume that $N(\alpha)$ is disjoint from the postcritical set of $f$. We let $\Delta_{\alpha_{i, j}}$ be a linearizable disk contained in $\bigcap_{\alpha \in \mathcal{C}^{\prime} \backslash\left\{\alpha_{i j}\right\}} f^{\ell(\alpha)}(N(\alpha))$. For any preimage $\alpha \neq \alpha_{i, j}$ of $\alpha_{i, j}$ (including those in $\mathcal{C}$ ), we let $\ell$ be the minimal iterate such that $f^{\ell}(\alpha)=\alpha_{i, j}$ as above, and we define $\Delta_{\alpha}$ to be the connected component of $f^{-\ell}\left(\Delta_{\alpha_{i, j}}\right)$ which contains $\alpha$.

Since $\beta_{i}$ is disjoint from the $\omega$-limit set of any recurrent critical point, it follows from R. Mañé's theorem $[14,23]$ that there are a linearizable disk $\Delta_{\beta_{i}}^{\prime}$ and an integer $p>0$ such that, for any iterate $n \geq 0$ and any connected component $W$ of $f^{-n}\left(\Delta_{\beta_{i}}^{\prime}\right)$, the degree of $\left.f^{n}\right|_{W}$ is at most $p$. We choose $\Delta_{\beta_{i}}^{\prime}$ to be such a disk centered at $\beta_{i}$ of a certain radius $r_{\beta_{i}}^{\prime}$.

Definition of $\mathcal{Y}^{*}, \mathcal{G}, \mathcal{G}^{*}$. Recall that $\mathcal{Y}$ is the grand orbit of $\bigcup_{\kappa} Y(\kappa)$. It is fully invariant, contains the support of $\sigma_{t}$ and $\mathcal{R} \backslash J_{f}$. Set $\mathcal{Y}^{*}=\mathcal{Y} \cup \mathcal{R}$. Each component of $\mathcal{Y}^{*}$ is star-like, and consists of finitely many $\mathcal{Y}$-components together with a common boundary point. We define $\mathcal{G}$ (for green) to be an open neighborhood of $\mathcal{Y}$ with the property that on $\mathcal{G} \backslash \mathcal{Y}, \sigma_{t}$ is conformal, and $f(\mathcal{G}) \subset \mathcal{G}$. For each attracting point $\alpha_{i, j}$, we let $\kappa_{i, j}$ be the edge in $\widehat{R}$ which joins $\alpha_{i, j}$ to the center $\beta_{i}$. Let $G_{i, j}=G^{\prime}\left(\kappa_{i, j}\right) \cup \frac{1}{2} \Delta_{\alpha_{i, j}}$. For any preimage $\kappa \neq$ $\kappa_{i, j}$ of $\kappa_{i, j}$, let $n$ be the first iterate such that $f^{n}(\kappa)=\kappa_{i, j}$. We define $G(\kappa)$ to be the connected component of $f^{-n}\left(G_{i, j}\right)$ which contains $\kappa$. Similarly, we define $\mathcal{G}^{*}=\mathcal{G} \cup \mathcal{R}$, which is also the union of $\mathcal{G}$ with the grand orbits of all the centers of the stars $\gamma_{i}$. Each $\mathcal{G}^{*}$-component is again star-like. It is important for what follows that $\mathcal{G}$ is open, whereas $\mathcal{G}^{*}$ is neither open nor closed.

Definition of $\mathcal{B}$. We define $\mathcal{B}$ (for blue) as the collection of the following sets. We set

$$
B\left(\gamma_{i}\right)=\left(\bigcup_{\kappa}\left(B^{\prime}(\kappa) \cup \Delta_{\alpha_{\kappa}}\right)\right) \cup \Delta_{\beta_{i}},
$$

where $\Delta_{\beta_{i}}$ is a disk centered at $\beta_{i}$ of radius $r_{\beta_{i}} / 2 \leq r_{\beta_{i}}^{\prime} / 6$, where $\kappa$ ranges over the edges attached to $\beta_{i}$, and $\alpha_{\kappa}$ is the attracting point attached to $\kappa$. The constant $r_{\beta_{i}}$ depends on $f$, and will be defined in Section 2.4 below in which we prove that any limit of $\left(f_{t}\right)$ is weakly hyperbolic. The collection $\mathcal{B}$ is defined as follows: for any connected component $R$ of $\mathcal{R}$, there is a minimal iterate such that $f^{n}(R)=\gamma$; we let $B(R)$ be the connected component of $f^{-n}(B(\gamma))$ which contains $R$. Note that $\left.\operatorname{deg} f^{n}\right|_{B(R)} \leq p$ by construction. Without loss of generality, we may assume that $p=\delta$. 
We use $G$ (resp. $\left.Y, R, G^{*}, Y^{*}\right)$ to denote a (general) $\mathcal{G}$ (resp. $\mathcal{Y}, \mathcal{R}$, $\left.\mathcal{G}^{*}, \mathcal{Y}^{*}\right)$-component. Each point $\beta \in \mathcal{R} \cap J_{f}$ is the center of a unique $R, Y^{*}$ and $G^{*}$, with $R \subset Y^{*} \subset G^{*}$, and they are compactly contained in a unique element $B$ of $\mathcal{B}$. For each $G$, denote by $\beta(G)$ the unique Julia point on $\partial G$ (it is a preimage of the central vertex $\beta_{i}$ of some $\gamma_{i}$ ).

Normalization. The point $a=\infty$ is a critical point which belongs to a periodic Fatou component of $f$, which always exists since $f$ has an attracting point. Its first return into the same Fatou component is $b=0$. The point $c=1$ is another point outside $\mathcal{G}^{*}$. Note that $a, b, c \notin \mathcal{G}^{*}$. This normalization has the advantage that we will be able to work with the Euclidean metric as well as with the spherical one, because then $\overline{J_{f} \cup \mathcal{B}} \subset \mathbb{C}$. If $K$ is a 1-neighborhood of $\overline{J_{f} \cup \mathcal{B}}$ in the Euclidean metric, then both metrics are equivalent on $K$, i.e., there is a constant $c_{s}>1$ such that, for all $x, y \in K$, $|x-y| / c_{s}<d(x, y)<c_{s}|x-y|$.

Lemma 2.2. (1) For each component $\gamma$ of $\mathcal{R}$, there is a $\mathcal{B}$-component $B(\gamma)$ containing $\gamma$ and an iterate $n(\gamma)$ such that $f^{n(\gamma)}(\gamma)$ is the periodic star chosen above and such that the degree of $\left.f^{n(\gamma)}\right|_{B(\gamma)}$ is at most $\delta$.

(2) The diameter of any sequence of distinct stars $G_{k}^{*}$ of $\mathcal{G}^{*}$ tends to 0.

Proof. The first statement follows from the construction of $\mathcal{B}$. Set $B_{k}=$ $B\left(G_{k}^{*}\right)$. There are (minimal) iterates $n_{k} \geq 0$ such that $f^{n_{k}}\left(B_{k}\right)=B(\gamma)$, with $n_{k} \rightarrow \infty$ as $k \rightarrow \infty$. Since $\left.\operatorname{deg} f^{n}\right|_{B_{k}} \leq \delta$ for all $k$, the lemma is a consequence of the so-called shrinking lemma (see p. 86 of [13] for a proof).

\subsection{Equicontinuity of $\left(h_{t}\right)$ and fiber structure of limit maps. In} this section we prove:

Proposition 2.3. In the setting of Theorem 2.1, the maps $\left(h_{t}\right)$ are equicontinuous. Furthermore, for any limit map $H$ of $\left(h_{t}\right)$, the nontrivial fibers of $H$ are exactly the $\mathcal{R}$-components.

For this we need

LEMma 2.4. The family $\left(h_{t}\right)$ is uniformly equicontinuous if and only if it is pointwise equicontinuous, i.e., for any $z_{0} \in \overline{\mathbb{C}}$ and any $\varepsilon>0$, there exist $\eta>0$ and $t_{0}<1$ such that for any $t \in\left[t_{0}, 1\right)$ and any $y$ with $d\left(y, z_{0}\right) \leq \eta$, we have $d\left(h_{t}(y), h_{t}\left(z_{0}\right)\right) \leq \varepsilon$ (where $d$ denotes the spherical metric).

The proof is the same as for the statement that a pointwise continuous map on a compact set is uniformly continuous.

Now the proof of Proposition 2.3 is decomposed into 4 steps.

STEP 1. Equicontinuity of $\left(h_{t}\right)$ at any $z_{0} \notin J_{f} \cup \mathcal{R}$. This follows from the local uniform quasiconformality due to the construction of $\widetilde{P}_{t}$. 
For the remaining cases the only information we have on the family $\left(h_{t}\right)$ is on its Beltrami forms, and therefore how conformal invariants are modified after the application of $h_{t}$. So the equicontinuity will be proved by using the following lemma which enables us to translate conformal invariants estimates into metric estimates.

Lemma 2.5 (equicontinuity criterion at a point). Let $\mathcal{A}=\{h: \mathbb{D} \rightarrow \mathbb{C}\}$ be a family of continuous injective maps such that $\bigcup_{h \in \mathcal{A}} h(\mathbb{D})$ avoids at least 2 points in $\mathbb{C}$.

(1) Let $\left(U_{n}\right)_{n \geq 0}$ be a nested sequence of disk-like neighborhoods of the origin in the unit disk $\mathbb{D}$ such that $A_{n}^{\prime}=\mathbb{D} \backslash \bar{U}_{n}$ is an annulus. If there exists a sequence $\eta_{n} \nearrow \infty$ such that

$$
\forall h \in \mathcal{A}, \forall n \geq 0, \quad \bmod h\left(A_{n}^{\prime}\right) \geq \eta_{n},
$$

then $\mathcal{A}$ is equicontinuous at the origin.

(2) Let $A_{n} \subset \mathbb{D}$ be a nested sequence of annuli (i.e. for all $n, A_{n+1}$ is contained in the component of $\mathbb{C} \backslash \bar{A}_{n}$ containing 0$)$. If there is $M>0$ such that

$$
\forall h \in \mathcal{A}, \forall n \geq 0, \quad \bmod h\left(A_{n}\right) \geq M,
$$

then $\mathcal{A}$ is equicontinuous at the origin.

The proof of (1) relies on the fact that if an annulus $A^{\prime}$ in $\overline{\mathbb{C}}$ has modulus at least $C$, then one of the complementary components of $A^{\prime}$ has spherical diameter at most $D$, where $D$ depends only on $C$. Refer for example to Theorem 2.4 of [16] or to [23, appendices A and B]. Part (2) follows by applying the Grötzsch inequality.

Lemma 2.6 (control of moduli of deformed annuli). Let $A \subset \mathbb{C}$ be $a$ bounded annulus such that $\partial A \cap \mathcal{G}=\emptyset$ (recall that $\mathcal{G}$ is open). Then there is $m>0$ (depending on $A$ but not on $t$ ) such that $\bmod h_{t}(A) \geq m$ for all $t$.

We postpone the proof of this lemma to $\S 2.3$.

Lemma 2.7 (one good annulus around each Julia point). Fix $r>0$ (which will be the constant for $f$ in the definition of weak hyperbolicity).

For any $x \in J_{f} \backslash \mathcal{R}$, there are two open neighborhoods $N^{\prime}(x)$ and $N(x)$ of $x$ in $D(x, r / 4)$ and $m>0$ such that $\bmod h_{t}\left(N(x) \backslash \overline{N^{\prime}(x)}\right) \geq m$ for all $t$.

For any $x=\beta_{\gamma} \in \mathcal{R}$, with $\gamma$ an $\mathcal{R}$-component with repelling end $\beta_{\gamma}$ and $B(\gamma)$ the corresponding $\mathcal{B}$-component, there are two open neighborhoods $N^{\prime}(\gamma)$ and $N(\gamma)$ of $\gamma$ in $\left(D\left(\beta_{\gamma}, r / 4\right) \cup \mathcal{F}\right) \cap B(\gamma)$, labeled also by $N\left(\beta_{\gamma}\right)$ and $N^{\prime}\left(\beta_{\gamma}\right)$, and there is $m>0$ such that $\bmod h_{t}\left(N\left(\beta_{\gamma}\right) \backslash \overline{N^{\prime}\left(\beta_{\gamma}\right)}\right) \geq m$ for all $t$.

Proof. Let $x \in J_{f} \backslash \mathcal{R}$. Choose Jordan neighborhoods $\overline{N^{\prime}} \subset N \subset$ $D(x, r / 4)$ of $x$ avoiding at least two marked points such that no $\mathcal{G}^{*}$-compo- 
nent would have a closure that intersects both boundaries. Then

$$
N^{\prime} \cup\left(\bigcup_{\substack{G \cap \partial N^{\prime} \neq \emptyset \\ G \text { a } \mathcal{G} \text {-comp. }}} \bar{G} \cup \partial N^{\prime}\right) \subset N \backslash\left(\bigcup_{\substack{G \cap \partial N \neq \emptyset \\ G \text { a } \mathcal{G} \text {-comp. }}} \bar{G} \cup \partial N\right) .
$$

Moreover the right hand set is open and the left hand set is compact connected. So their difference has an annular component $A$ satisfying $\partial A \cap \mathcal{G}$ $=\emptyset$.

There is $m>0$ such that

$$
\bmod h_{t}\left(N \backslash \overline{N^{\prime}}\right) \stackrel{\text { Grötzsch }}{\geq} \bmod h_{t}(A) \stackrel{\text { Lemma } 2.6}{\geq} m .
$$

Let $x=\beta \in J_{f} \cap \mathcal{R}$. We will choose $N$ and $N^{\prime}$ similarly, but as neighborhoods of $\overline{G^{*}}(\beta)$ and as subsets of $(\mathcal{B}(x) \cap \mathcal{F}) \cup D(x, r / 4)$.

Now we can prove

STEP 2. Equicontinuity of $\left(h_{t}\right)$ at $z_{0} \in J_{f} \backslash(\mathcal{R} \cup\{$ preparabolics $\})$.

Proof. We will use a standard pullback argument. When $x$ ranges over $J_{f}$, the sets $N^{\prime}(x)$ define an open cover of $J_{f} \cup \mathcal{R}$, which is compact. We extract a finite subcovering $N^{\prime}\left(x_{i}\right), i=1, \ldots, l$. Note that Lemma $2.7 \mathrm{im}-$ plies the existence of $m>0$ such that, for any $t$ and any $i \in\{1, \ldots, l\}$, $\bmod h_{t}\left(N\left(x_{i}\right) \backslash \overline{N^{\prime}\left(x_{i}\right)}\right) \geq m$.

Assume $z_{0} \in J_{f} \backslash(\mathcal{R} \cup\{$ preparabolics $\})$. By weak hyperbolicity, there are infinitely many $n$ (the good iterates) such that $f^{n}$ blows up a neighborhood of $z_{0}$ to $D\left(f^{n}\left(z_{0}\right), r\right)$ with degree at most $\delta$. There is $i(n)$ such that $f^{n}\left(z_{0}\right) \in$ $N^{\prime}\left(x_{i(n)}\right)$. Taking a subsequence if necessary we may assume $i(n) \equiv i$.

We distinguish two cases. Either $x_{i}=\beta_{\gamma}$ for some $\gamma$; then we use the fact that $N\left(x_{i}\right)$ is contained in a $\mathcal{B}$-component so that we may apply Lemma 2.2. Or

$$
f^{n}\left(z_{0}\right) \in N^{\prime}\left(x_{i}\right) \subset N\left(x_{i}\right) \subset D\left(x_{i}, \frac{r}{4}\right) \subset D\left(f^{n}\left(z_{0}\right), \frac{r}{2}\right) \subset D\left(f^{n}\left(z_{0}\right), r\right)
$$

for infinitely many $n$. Let $E, U$, with $E \subset U$, be the respective components of $f^{-n}\left(\overline{N_{i}^{\prime}}\right)$ and of $f^{-n}\left(N_{i}\right)$ containing $z_{0}$. Set $A_{n}=U \backslash E$. Then

$$
\bmod h_{t}\left(A_{n}\right) \geq \frac{1}{\delta} \bmod h_{t}\left(N\left(x_{i}\right) \backslash \overline{N^{\prime}\left(x_{i}\right)}\right) \geq \frac{m}{\delta}
$$

(for the first inequality, see for example [23, the proof of Lemma 2.1]). Taking again a subsequence if necessary, we may assume that the annuli $A_{n}$ are disjoint, nesting down to $z_{0}$ (this follows from the shrinking lemma, due to the "Koebe space" $D(r) \backslash D(r / 2))$. This shows that $\left(h_{t}\right)$ is equicontinuous at $z_{0}$.

STEP 3. Equicontinuity of $\left(h_{t}\right)$ at $z_{0} \in \mathcal{R} \cup\{$ preparabolics $\}$. This part is postponed to $\S 2.5$. 
In order to study the fiber structure of limit maps of $\left(h_{t}\right)$, we will make use of the following lemma which is proved in $\S 2.3$ :

LEMMA 2.8 (4 points). Let $\left(z_{1}, z_{2}, z_{3}, z_{4}\right)$ be four distinct points such that no two belong to the same $\mathcal{R}$-component. Then, for $\Gamma$ the set of Jordan curves which separate $\left(z_{1}, z_{2}\right)$ from $\left(z_{3}, z_{4}\right)$, there is $m>0$ such that $\Lambda\left(h_{t}(\Gamma)\right) \geq m$ (for all $t$, where $\Lambda(\Gamma)$ denotes the extremal length of the curve family $\Gamma$, cf. [2])).

STEP 4. For any limit map of $\left(h_{t}\right)$, the nontrivial fibers are $\mathcal{R}$-components.

Proof. Recall that $\left(h_{t}\right)$ are normalized to fix three points $a, b, c$ in the complement of $\mathcal{G}^{*}$.

Assume $h_{t_{n}} \rightrightarrows H$. By Step 3 and its proof, $H$ maps each $\mathcal{R}$-component to a point. We will show that they are the only fibers of $H$.

Choose $z \neq w$, so that $z, w$ are not in the same $\mathcal{R}$-component. Assume that $H(z)=H(w)$. We may assume that $H(z) \notin\{a, b\}$ and that $\{z, w, a, b\}$ are in different $\mathcal{R}$-components by relabeling the points $a, b, c$ if necessary. Let $\Gamma_{(z, w),(a, b)}$ be the set of curves separating $\{z, w\}$ from $\{a, b\}$. The assumptions of Lemma 2.8 are satisfied, so $\Lambda\left(h_{t}\left(\Gamma_{(z, w),(a, b)}\right)\right) \geq m>0$ for all $t$. Consequently, $H(z) \neq H(w)$ (see Corollary B.2 in the appendix).

This ends the proof of Proposition 2.3, modulo Lemma 2.6, Lemma 2.8 and Step 3.

2.3. Estimates of conformal invariants. The technical Lemma 2.6 and Lemma 2.8 used above are proved in this section. The main idea is to formalize the noninfluence of the deformation in appropriate cases by "forgetting" its support.

We start with the key lemma due to Cui.

KEY LEMMA. There is a uniform constant $0<c \leq 1$ with the following properties. Let $\eta:[0,1] \rightarrow \mathbb{C}$ (resp. $\eta:[0,1] \rightarrow \overline{\mathbb{C}})$ be a rectifiable curve with end points outside $\mathcal{G}$. Then

$$
l_{\varrho_{e}}(\eta) \geq c d_{e}(\eta(0), \eta(1)) \quad\left(\operatorname{resp} . l_{\varrho}(\eta) \geq c d(\eta(0), \eta(1))\right),
$$

where $d_{e}$ is the Euclidean metric (resp. $d$ the spherical metric), $\varrho_{e}$ (resp. @) is the same metric but with zero density in $\mathcal{Y}$ (the support of $\left.\sigma_{t}\right)$, i.e.

$$
\varrho_{e}(z)|d z|=(1-\chi \mathcal{Y}(z))|d z|, \quad \varrho(z)|d z|=\frac{1-\chi \mathcal{Y}(z)}{1+|z|^{2}}|d z|,
$$

where $\chi \mathcal{Y}$ denotes the indicatrix function associated to $\mathcal{Y}$.

Proof. We will modify $\eta$ in the following way that will also be used later on: Let $I$ be a maximal open subinterval such that $\eta(I) \subset \mathcal{G}$ (so that $\eta(I) \subset G$ and $\eta(\partial I) \subset \partial G$ for some $G)$. Define $\eta^{\prime}(I)$ to be the Euclidean 
(spherical) geodesic linking the two ends of $\eta(I)$. We then claim: for some $c>0$,

$$
l_{\varrho_{e}}(\eta(I)) \geq c l_{e}\left(\eta^{\prime}(I)\right) \quad\left(\operatorname{resp} . l_{\varrho}(\eta(I)) \geq c l\left(\eta^{\prime}(I)\right)\right) .
$$

Proof of the claim: If $\eta(I) \cap \mathcal{Y}=\emptyset$, then $l_{\varrho_{e}}(\eta(I))=l_{e}(\eta(I)) \geq l_{e}\left(\eta^{\prime}(I)\right)$. If $\eta(I) \cap \mathcal{Y} \neq \emptyset$, set $I=] s_{1}, s_{2}[$; then there are two subintervals, one on each end, $\left.I_{1}=\right] s_{1}, s_{1}^{\prime}\left[\right.$ and $\left.I_{2}=\right] s_{2}^{\prime}, s_{2}\left[\right.$, such that $\eta\left(s_{i}\right) \in \partial G$ and $\eta\left(s_{i}^{\prime}\right) \in \partial(\mathcal{Y} \cap G)$. We claim that there are constants $c_{G}, c>0$, with $c$ independent of $G$, such that

$$
\begin{aligned}
l_{\varrho_{e}}(\eta(I)) & \geq \sum_{i=1,2} l_{\varrho_{e}}\left(\eta\left(I_{i}\right)\right)=\sum_{i=1,2} l_{e}\left(\eta\left(I_{i}\right)\right) \geq \sum_{i=1,2}\left|\eta\left(s_{i}\right)-\eta\left(s_{i}^{\prime}\right)\right| \\
& \stackrel{*}{\geq} c_{G} \sum_{i=1,2}\left|\eta\left(s_{i}\right)-\beta(G)\right| \geq c_{G}\left|\eta\left(s_{1}\right)-\eta\left(s_{2}\right)\right| \\
& =c_{G} l_{e}\left(\eta^{\prime}(I)\right) \stackrel{*}{\geq} c l_{e}\left(\eta^{\prime}(I)\right) .
\end{aligned}
$$

Here the only nontrivial inequalities are the ones marked with $*$. Lemma 2.9 below proves the first $*$-inequality.

LEMma 2.9. If $g: \mathbb{D} \rightarrow \mathbb{C}$ is a univalent function such that $g(z)=$ $\lambda z+O(z)$ at the origin with $|\lambda|>1$, and if $\gamma_{1}$ and $\gamma_{2}$ are two disjoint invariant arcs in $\mathbb{D} \backslash\{0\}$ landing at 0 , then $\gamma_{1} \cup \gamma_{2}$ forms a "quasi-arc" in the following sense: there is a constant $c>0$, depending only on the germ $g$, such that for any $z \in \gamma_{2}, d_{e}\left(z, \gamma_{1}\right) \geq c d_{e}(z, 0)$.

Proof. Let $\phi: \bar{U} \rightarrow \bar{D}_{r}$ be a linearization mapping (with $\bar{U} \subset \mathbb{D}$ so that $\phi(\bar{U})$ is a round disk). As $\left|\phi^{\prime}(z)\right|$ is bounded from above and from below, we may estimate the distance in the $\phi(z)$ coordinate. For $\lambda=g^{\prime}(0), \phi\left(\gamma_{1}\right)$ and $\phi\left(\gamma_{2}\right)$ are both invariant under $z \mapsto \lambda z$. In other words, they are self-similar. There is a constant $c_{0}>0$ such that for $z \in \phi\left(\gamma_{2}\right) \cap\{r /|\lambda| \leq|w| \leq r\}$, $d\left(z, \phi\left(\gamma_{1}\right)\right) \geq c_{0}|z|$, due to compactness and the fact that the two arcs are disjoint. The rest follows by self-similarity and bounded distortion of the univalent map $\phi$.

For each $\mathcal{G}$-component $G$ there is a minimal $n$ such that $f^{n}(\beta(G))$ is $q$-periodic and repelling, and $f^{n}$ is either locally injective at $\beta(G)$ or has an isolated critical point at $\beta(G)$. We may then apply the above lemma to the (well defined) map $F=f^{-n} f^{q} f^{n}$ in a neighborhood $U_{G}$ of $\beta(G)$. Combining this with a compactness argument for $\bar{G} \backslash U_{G}$, we obtain the constant $c_{G}$.

It remains to show the second starred inequality, that is, $c=\inf _{G} c_{G}>0$.

Assume by contradiction that $\inf _{G} c_{G}=0$. Then for some periodic $\gamma$ and its corresponding $\mathcal{B}$-component $B$, there are $n_{k} \rightarrow \infty, B_{k} \in \mathcal{B}$, a $\mathcal{G}$ component $G_{k}$ in $B_{k}$ with attaching point $\beta_{k}$, and $z_{k} \in \partial G_{k} \backslash\left\{\beta_{k}\right\}, z_{k}^{\prime} \in$ $G_{k} \cap \partial \mathcal{Y}$ such that $\left|z_{k}-z_{k}^{\prime}\right| /\left|z_{k}-\beta_{k}\right| \rightarrow 0$ as $k \rightarrow \infty$. 


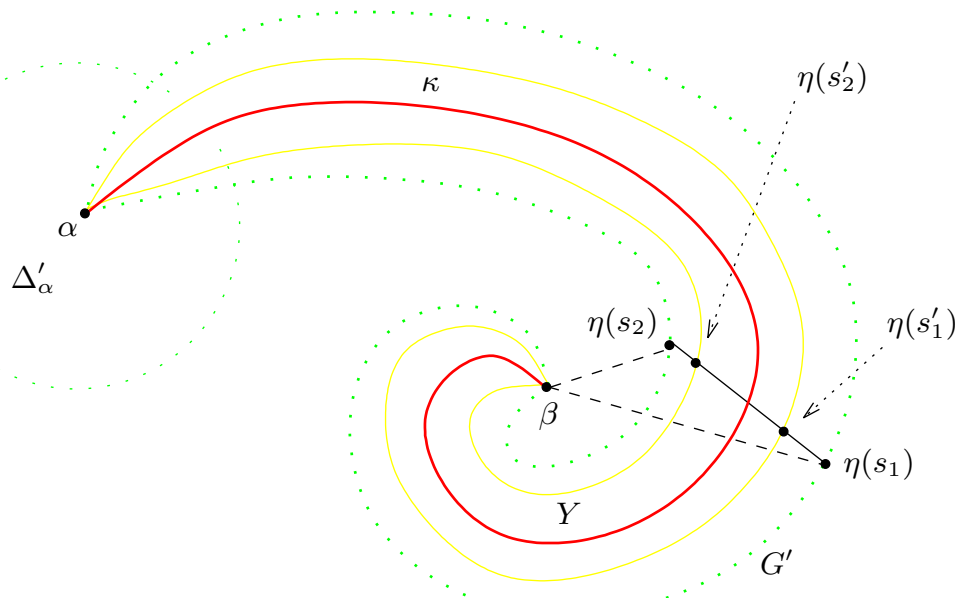

Fig. 3. Cui's inequality

We let $\widehat{G}$ denote the union of the $\mathcal{G}$-components attached to $\beta=\beta(\gamma)$ (i.e. $\left.\widehat{G}=G^{*}(\beta) \backslash\{\beta\}\right)$. Set $y_{k}=f^{n_{k}}\left(z_{k}\right), y_{k}^{\prime}=f^{n_{k}}\left(z_{k}^{\prime}\right)$. We have $\beta=f^{n_{k}}\left(\beta_{k}\right)$ and it follows from the above that $\left|y_{k}-y_{k}^{\prime}\right| /\left|y_{k}-\beta\right| \geq C^{\prime}>0$. We will uniformize the domains in order to deal with proper maps $\psi_{k}$ of the unit disk of degree at most $\delta$ :

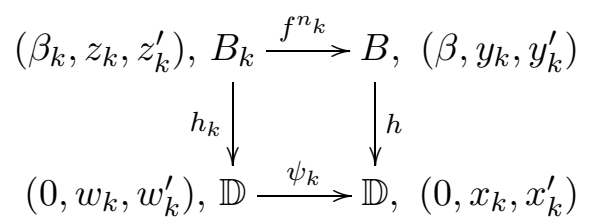

Since $h$ has bounded distortion on $\widehat{G}$ which contains $y_{k}, y_{k}^{\prime}$, we have $\left|x_{k}-x_{k}^{\prime}\right| /\left|x_{k}\right| \geq C>0$ and $h(\widehat{G}) \subset \bar{D}(0, r)$ for some $r<1$. As $w_{k}, w_{k}^{\prime}, 0$ are in the same component of $\psi_{k}^{-1}(h(\widehat{G}))$, we know that $\left|w_{k}\right|,\left|w_{k}^{\prime}\right| \leq s<1$ (see e.g. [23, Lemma 2.1]). Therefore $h_{k}^{-1}$ has uniform bounded distortion, so that $\left|w_{k}-w_{k}^{\prime}\right| /\left|w_{k}\right|$ is comparable to $\left|z_{k}-z_{k}^{\prime}\right| /\left|z_{k}-\beta_{k}\right|$ and tends to 0 .

CASE 1: $\left|x_{k}\right| \geq r_{1}>0$. This implies that $\left|x_{k}-x_{k}^{\prime}\right| \geq C r_{1}>0$. Switch to the hyperbolic metric $\varrho(\cdot, \cdot)$ for convenience, and use the fact that $\psi_{k}$ contracts $\varrho$; we have

$$
\frac{\varrho\left(w_{k}, w_{k}^{\prime}\right)}{\varrho\left(w_{k}, 0\right)} \geq \frac{\varrho\left(x_{k}, x_{k}^{\prime}\right)}{C_{1}} \geq C_{2}>0
$$

which is a contradiction.

CASE 2: $\left|x_{k}\right| \rightarrow 0$. One can check that $w_{k}$ and 0 are in the same component of $\psi_{k}^{-1}\left(D\left(0,2\left|x_{k}\right|\right)\right)$. This implies that $\varrho\left(w_{k}, 0\right) \rightarrow 0$ (see for example [23, Lemma 2.1]); therefore $\varrho\left(w_{k}, w_{k}^{\prime}\right) \rightarrow 0$ and $\varrho\left(x_{k}, x_{k}^{\prime}\right) \rightarrow 0$. 
We claim that there is $a>0$ such that $\left|x_{k}^{\prime}\right| \leq a\left|x_{k}\right|$. For otherwise for infinitely many $k, \psi_{k}^{-1}\left(\left\{z:\left|x_{k}\right|<|z|<\left|x_{k}^{\prime}\right|\right\}\right)$ would contain a round annulus centered at 0 , separating $w_{k}$ from $w_{k}^{\prime}$, and having definite modulus. This contradicts the fact that $\left|w_{k}-w_{k}^{\prime}\right| /\left|w_{k}\right| \rightarrow 0$.

For all large $k$, set $W_{k}=D\left(0,2 a\left|x_{k}\right|\right) \subset \mathbb{D}$ and let $W_{k}^{\prime}$ be the component of $\psi^{-1}\left(W_{k}\right)$ containing 0 . One checks again that $w_{k}, w_{k}^{\prime} \in W_{k}^{\prime}$. Now uniformizing $W_{k}, W_{k}^{\prime}$ again we are more or less back in the situation of Case 1 (by checking as well that $w_{k}, w_{k}^{\prime}, 0$ are always in the same pulled-back components). Therefore $\inf _{G} c_{G}>0$.

This ends the proof of the claim for $l_{e}$. The spherical case is similar. We then get the Key Lemma by replacing $\eta(I)$ by $\eta^{\prime}(I)$ for every possible $I$.

Proof of Lemma 2.6 (control of moduli of deformed annuli). Denote by $\delta$ the Euclidean distance between the two components of $\partial A$, and let $\Gamma$ be the family of rectifiable curves joining them. It follows from the Key Lemma that for any $\eta \in \Gamma, l_{\varrho_{e}}(\eta) \geq c \delta$. Set $A_{t}=h_{t}(A)$, and let $\Gamma_{t}$ be the family of arcs joining the two boundary components of $A_{t}$, and $\varrho_{t}=h_{t *}(\varrho)$ (with $\varrho_{t}=0$ on $\left.h_{t}(\mathcal{Y})\right)$. Then

$$
\begin{aligned}
\bmod A_{t} & =\Lambda\left(\Gamma_{t}\right)=\sup _{\varrho^{\prime}} \frac{L_{\varrho^{\prime}}\left(\Gamma_{t}\right)^{2}}{\operatorname{Area}_{\varrho^{\prime}} A_{t}} \geq \frac{L_{\varrho t}\left(\Gamma_{t}\right)^{2}}{\operatorname{Area}_{\varrho t} A_{t}}=\frac{L_{\varrho}(\Gamma)^{2}}{\operatorname{Area}_{\varrho} A} \\
& \geq \frac{(c \delta)^{2}}{\operatorname{Area}_{\varrho} A} \geq \frac{(c \delta)^{2}}{\operatorname{Area}(A)}=: m(A)>0,
\end{aligned}
$$

where the supremum is taken over all measurable conformal metrics $\varrho^{\prime}$ on $A_{t}$, and $L_{\varrho^{\prime}}\left(\Gamma_{t}\right)$ denotes the infimum of the $\varrho^{\prime}$-lengths of curves in $\Gamma_{t}$.

Before proving Lemma 2.8, we first establish a lemma that gives a uniform control. This estimate will also be used to prove the weak hyperbolicity of limit maps of $\left(f_{t}\right)$.

Let $z \in \overline{\mathbb{C}}$. We assign to $z$ a compact subset $K(z)$ in the following way. If $z \in \mathcal{G}^{*}$, then there is a unique $\mathcal{G}^{*}$-component $G^{*}$ and a unique $\mathcal{Y}^{*}$-component $Y^{*}$ such that $z \in G^{*}$ and $Y^{*} \subset G^{*}$. We set $K(z)=Y^{*}$. If $z \notin \mathcal{G}^{*}$, we set $K(z)=\{z\}$.

Let $\mathcal{Q}$ be the set of distinct quadruples $q=\left(z_{1}, z_{2}, z_{3}, z_{4}\right)\left(z_{i} \neq z_{j}\right.$ for $i \neq j$ ). If $q \in \mathcal{Q}$, we let $\Gamma_{q}$ be the set of rectifiable curves which separate $\left(z_{1}, z_{2}\right)$ from $\left(z_{3}, z_{4}\right)$.

For $r>0$, we define $\mathcal{Q}_{r} \subset \mathcal{Q}$ as the set of quadruples such that $d\left(K\left(z_{1}\right), K\left(z_{2}\right)\right) \geq r, d\left(K\left(z_{3}\right), K\left(z_{4}\right)\right) \geq r, d\left(z_{1}, z_{2}\right) \geq r$ and $d\left(z_{3}, z_{4}\right) \geq r$.

Lemma 2.10 (uniform control of lengths). For all $r>0$, there is a constant $\ell=\ell(r)>0$ such that, for any $q \in \mathcal{Q}_{r}$ and any $\gamma \in \Gamma_{q}$, we have $l_{\varrho}(\gamma) \geq \ell\left(\right.$ where $l_{\varrho}$ is given in the Key Lemma). 
Proof. If not, let $q_{n} \in \mathcal{Q}_{r}$ and $\gamma_{n} \in \Gamma_{q_{n}}$ be such that $l_{\varrho}\left(\gamma_{n}\right) \rightarrow 0$. Taking subsequences if necessary, we may assume that $q_{n}$ tends towards $\left(z_{1}, z_{2}, z_{3}, z_{4}\right) \in \overline{\mathbb{C}}^{4}$, and that $\gamma_{n}$ tends in the Hausdorff topology towards a compact subset $\gamma$ of diameter at least $r$.

Let $\gamma_{n}^{\prime}=\gamma_{n} \backslash \mathcal{G}$. If there is a subsequence such that $\operatorname{diam}\left(\gamma_{n_{k}}^{\prime}\right) \geq \delta>0$ for all $k \geq 0$, then it follows from the Key Lemma that

$$
l_{\varrho}\left(\gamma_{n_{k}}\right) \geq 2 c \operatorname{diam}\left(\gamma_{n_{k}}^{\prime}\right) \geq 2 c \delta>0,
$$

which is a contradiction.

Therefore, $\operatorname{diam}\left(\gamma_{n}^{\prime}\right) \rightarrow 0$. Taking a further subsequence we may also assume that $\gamma_{n}^{\prime} \rightarrow\{a\}$ in the Hausdorff topology. As $\mathcal{G}$ is open, we have $a \notin \mathcal{G}$. Therefore, for any $s$ with $0<s<r$, and for all large $n$, we have

$$
\gamma_{n}^{\prime} \subset D(a, s), \quad \gamma_{n} \subset D(a, s) \cup \mathcal{G}^{*} .
$$

Hence $\gamma_{n}$ is contained in the connected component $D_{s}^{\prime}$ of $D(a, s) \cup \mathcal{G}^{*}$ containing $a$.

CASE 1: $a \notin \overline{G^{*}}$ for any $\mathcal{G}^{*}$-component $G^{*}$. Then $\operatorname{diam} D_{s}^{\prime} \rightarrow 0$ as $s \rightarrow 0$. This contradicts diam $\gamma_{n} \geq r$.

CASE 2: $a \in \partial G^{*} \backslash\left\{\beta\left(G^{*}\right)\right\}$ for some $\mathcal{G}^{*}$-component $G^{*}$. Then for $s$ small enough, and $n$ large, $\gamma_{n} \subset D_{s}^{\prime}=D(a, s) \cup G^{*}$. Hence a pair of points in the quadruple $q_{n}$, say $z_{1 n}, z_{2 n}$, are in $D(a, s) \cup G^{*}$. But $q_{n} \in \mathcal{Q}_{r}$. This implies that $\left\{z_{1 n}, z_{2 n}\right\} \not \subset D(a, s)$, and $\left\{z_{1 n}, z_{2 n}\right\} \not \subset G^{*}$. Therefore, say, $z_{1 n} \in G^{*}$ and $z_{2 n} \in D(a, s)$, and consequently $z_{1} \in \overline{G^{*}} \backslash D(a, s)$ and $z_{2}=a$. It follows that $l_{\varrho}(\gamma) \geq \min \left\{d(a, \mathcal{Y}), d\left(a, z_{1}\right)\right\}>0$, which is a contradiction.

CASE 3: $a=\beta\left(G^{*}\right)$ for some $\mathcal{G}^{*}$-component $G^{*}$. In this case we have $\operatorname{diam}\left(D_{s}^{\prime} \backslash \overline{G^{*}}\right) \rightarrow_{s \rightarrow 0} 0$. It is then easy to see that $d\left(K\left(z_{1 n}\right), K\left(z_{2 n}\right)\right) \rightarrow 0$. This gives us a contradiction.

Proof of Lemma 2.8 (4 points). We proceed as in Lemma 2.6 but with the spherical metric. If neither $\left\{z_{1}, z_{2}\right\}$ nor $\left\{z_{3}, z_{4}\right\}$ is a subset of a green star of $\mathcal{G}^{*}$, then $q=\left(z_{1}, z_{2}, z_{3}, z_{4}\right)$ belongs to some $\mathcal{Q}_{r}$. Therefore, Lemma 2.10 implies that $l_{\varrho}(\gamma) \geq \ell>0$ for all $\gamma \in \Gamma_{q}$ and for some $\ell=\ell(r)$, and we obtain

$$
\Lambda\left(h_{t}(\Gamma)\right) \geq \frac{\ell^{2}}{4 \pi}>0 .
$$

Otherwise, $z_{1}$ and $z_{2}$, say, belong to the same star $G^{*}$ of $\mathcal{G}^{*}$. By assumption, at least one point in each group is not in $\mathcal{R}$. We now consider a new, smaller pair $\left(\widehat{\mathcal{Y}}^{*}, \widehat{\mathcal{G}}^{*}\right)$ of neighborhoods of $\mathcal{R} \backslash J_{f}$ such that neither $\left\{z_{1}, z_{2}\right\}$ nor $\left\{z_{3}, z_{4}\right\}$ is a subset of a $\widehat{\mathcal{G}}^{*}$-component. Now we will need our technical assumption on the complex structure $\sigma_{t}(z)$. It implies that there is $\left.t_{0} \in\right] 0,1[$ such that $\sigma_{s}=\sigma_{t_{0}}$ off $\widehat{\mathcal{Y}}^{*}$ for all $t_{0}<s<1$. Therefore $h_{s} \circ h_{t_{0}}^{-1}$ is conformal off $h_{t_{0}}\left(\widehat{\mathcal{Y}}^{*}\right)$. On the other hand, we may check that the proofs of Key Lemma 
and Lemma 2.10 are still valid on $h_{t_{0}}(\overline{\mathbb{C}})$, for the dynamics of $f_{t_{0}}$, and for the pair $\left(h_{t_{0}}\left(\widehat{\mathcal{Y}}^{*}\right), h_{t_{0}}\left(\widehat{\mathcal{G}}^{*}\right)\right)$ (but probably with another constant $c$ ). Therefore, for all $t_{0}<s<1$,

$$
\Lambda\left(h_{s} h_{t_{0}}^{-1}\left(h_{t_{0}}(\Gamma)\right)\right) \geq \frac{\widehat{\ell}^{2}}{4 \pi}>0 .
$$

This implies that $\Lambda\left(h_{s}(\Gamma)\right) \geq C>0$ for $t_{0}<s<1$, with $C$ independent of $s$. But $\left(h_{t}\right)_{0 \leq t \leq t_{0}}$ is uniformly quasiconformal, so $\Lambda\left(h_{t}(\Gamma)\right) \geq m>0$ for all $t \in[0,1[$, with $m$ independent of $t$.

2.4. Equicontinuity of $\left(f_{t}\right)$ and the proof of Theorem 2.1. In this section, we prove the equicontinuity of $\left(f_{t}\right)_{t}$ together with the weak hyperbolicity of any of its limits, and then Theorem 2.1.

The equicontinuity of $\left(f_{t}\right)$ is due to Lemma A.4 in the appendix: in our case, assume $h_{t_{n}} \rightrightarrows H$. Then each $\mathcal{R}$-component is a fiber of $H$ and all the other fibers are points. Therefore $f$ maps any fiber of $H$ into a fiber of $H$. Replacing both $F_{t}$ and $G_{t}$ by $h_{t}$, and replacing $g$ by $f$ in Lemma A.4, we conclude that $f_{t_{n}}=h_{t_{n}} \circ f \circ h_{t_{n}}^{-1}$ converges uniformly to a limit map $F$. It is automatically a rational map of the same degree as $f$.

Let us prove that $F$ is weakly hyperbolic. Let $C=\inf _{t} \operatorname{dist}_{e}\left(0, J_{f_{t}}\right)$. Due to the facts that 0 is fixed in the Fatou set and disjoint from $J_{f} \cup \mathcal{R}$, and that $\left\{h_{t}\right\}_{t}$ is equicontinuous, we have $C>0$.

LEMMA 2.11. If $r<C / 2$ then there is some constant $r^{\prime}>0$ such that, for all $x \in J_{f} \backslash(\mathcal{R} \cup\{$ preparabolics $\})$ and any $y \notin \mathcal{G}^{*} \cup D(x, r)$, we have $|H(x)-H(y)| \geq r^{\prime}$.

Proof. We use the notation of $\S 2.3$. Let $x \in J_{f} \backslash(\mathcal{R} \cup\{$ preparabolics $\})$ and $y \in \mathbb{C} \backslash \mathcal{G}^{*}$ with $|y-x| \geq r$. Then $K(x)=\{x\}$ and $K(y)=\{y\}$. Let $\Gamma$ be the set of rectifiable curves which separate $\{x, y\}$ from $\{0, \infty\}$. The quadruple belongs to $\mathcal{Q}_{r}$. Lemma 2.10 implies that $\Lambda\left(h_{t}(\Gamma)\right) \geq C_{r}^{\prime}$ for all $t<1$. We claim that $\left|h_{t}(x)-h_{t}(y)\right| \geq C \exp \left(-2 \pi / C_{r}^{\prime}\right)$. This would imply the lemma.

If $\left|h_{t}(x)-h_{t}(y)\right| \geq C$ then the claim is proved. Otherwise, $\mid h_{t}(x)-$ $h_{t}(y)|<| h_{t}(x) \mid$, and therefore Lemma B.1 in the appendix implies that

$$
\left|h_{t}(x)-h_{t}(y)\right| \geq\left|h_{t}(x)\right| \exp \left(-2 \pi / C_{r}^{\prime}\right) \geq C \exp \left(-2 \pi / C_{r}^{\prime}\right),
$$

which is the claim.

Proof that $F$ is weakly hyperbolic. We assume that $f$ is weakly hyperbolic with constants $\left(\delta, r_{0}\right)$. The weak hyperbolicity will come from two different arguments. The first will follow from Lemma 2.11 when the point considered is far from $\mathcal{G}^{*}$-components with large diameter, the second from Lemma 2.2. We will use the notation introduced in $\S 2.1$. 
Given $n_{0} \in \mathbb{N}$, we let $\mathcal{G}^{*}\left(n_{0}\right)$ be the union of the $\mathcal{G}^{*}$-components $G^{*}$ such that there is an iterate $n \leq n_{0}$ such that $f^{n}\left(G^{*}\right)$ is the periodic $G_{i}$ chosen in the cycle. Since the diameters of the connected components of $\mathcal{G}^{*}$ shrink to 0 , we may choose $n_{0}$ large enough so that the diameter of any $\mathcal{G}^{*} \backslash \mathcal{G}^{*}\left(n_{0}\right)$-component does not exceed $\min \left\{r_{0}, r_{\beta_{i}}^{\prime}\right\} / 3$.

\section{Define}

$$
r_{\beta_{i}}=\min \left\{r_{0} / 3, d\left(\beta_{i}, \mathcal{G}^{*}\left(n_{0}\right) \backslash G^{*}\left(\beta_{i}\right)\right), r_{\beta_{i}}^{\prime} / 3\right\} .
$$

Recall that $B_{\beta_{i}}=B_{\beta_{i}}^{\prime} \cup D\left(\beta_{i}, r_{\beta_{i}} / 2\right)$. We let $r_{1}=\min d\left(G^{*}, \partial B\right)$ where $G^{*}$ ranges over $\mathcal{G}^{*}\left(n_{0}\right)$-components and $B=B_{G^{*}} \in \mathcal{B}$ satisfies $G^{*} \subset B$. We set $A\left(\beta_{i}\right)=\Delta_{\beta_{i}} \backslash f^{-k_{i}}\left(\Delta_{\beta_{i}}\right)$. There is an $r_{2}>0$ such that, for any $z \in J_{f} \cap A\left(\beta_{i}\right)$, the disk $D\left(z, r_{2}\right)$ is disjoint from $\mathcal{G}^{*}\left(n_{0}\right)$. Note that $r_{2} \leq\left|z-\beta_{i}\right| \leq r_{\beta_{i}} / 2$.

Let $r<\min \left\{C / 2, r_{0} / 3, r_{1} / 2, r_{2}\right\}$, and consider a point $x \in J_{f} \backslash(\mathcal{R} \cup$ \{preparabolics\}). There is a sequence $\left(n_{k}\right)$ such that $\operatorname{deg}\left(f^{n_{k}}: W_{k}(x) \rightarrow\right.$ $\left.D\left(f^{n_{k}}(x), r_{0}\right)\right) \leq \delta$. We construct inductively a sequence $\left(n_{p}^{\prime}\right)$ such that $\operatorname{deg}\left(F^{n_{p}^{\prime}}: W_{p}^{\prime}(H(x)) \rightarrow D\left(F^{n_{p}}(H(x)), r^{\prime}\right)\right) \leq \delta$, where $r^{\prime}$ is associated to $r$ by Lemma 2.11 .

We assume that we have already constructed $n_{1}^{\prime}, \ldots, n_{p-1}^{\prime}$. Let $k$ be the smallest index so that $n_{k}>n_{p-1}^{\prime}$. We distinguish two cases.

If $D\left(f^{n_{k}}(x), r\right) \cap \mathcal{G}^{*}\left(n_{0}\right)=\emptyset$, then we define $D_{k}^{\prime}$ to be the union of $D\left(f^{n_{k}}(x), r\right)$ with all $\mathcal{G}^{*}$-components $G^{*}$ such that $G^{*} \cap D\left(f^{n_{k}}(x), r\right) \neq \emptyset$. Since $r<r_{0} / 3$, it follows that $D_{k}^{\prime} \subset D\left(f^{n_{k}}(x), r_{0}\right)$ so it is also the case for the fill-in $D_{k}$ of $D_{k}^{\prime}$. It follows from Lemma 2.11 that $H\left(D_{k}\right) \supset D\left(H\left(f^{n_{k}}(x), r^{\prime}\right)\right)$. Let $W_{k}(H(x))$ be the connected component of $F^{-n_{k}}\left(D\left(F^{n_{k}}(H(x)), r^{\prime}\right)\right)$ which contains $H(x)$. Then the degree is at most $\delta$ since $D_{k} \subset D\left(f^{n_{k}}(x), r_{0}\right)$. Therefore, we set $n_{p}^{\prime}=n_{k}$.

If $D\left(f^{n_{k}}(x), r\right) \cap \mathcal{G}^{*}\left(n_{0}\right) \neq \emptyset$, then there is a $\mathcal{G}^{*}\left(n_{0}\right)$-component $G^{*}$ such that $f^{n_{k}}(x) \in B=B_{G^{*}}$ because $r<r_{1}$. Therefore, there is a minimal iterate $j$ such that $f^{n_{k}+j}(x) \subset A(\beta)$. We set $n_{p}^{\prime}=n_{k}+j$. Since $r<r_{2}$, it follows that $D\left(f^{n_{p}^{\prime}}(x), r\right) \cap \mathcal{G}^{*}\left(n_{0}\right)=\emptyset$; we let $D^{\prime}$ be the union of $D\left(f^{n_{p}^{\prime}}(x), r\right)$ with all $\mathcal{G}^{*}$-components which intersect that disk. Let $w \in D\left(f^{n_{p}^{\prime}}(x), r\right)$. Then

$$
\begin{aligned}
|w-\beta| & \leq\left|w-f^{n_{p}^{\prime}}(x)\right|+\left|f^{n_{p}^{\prime}}(x)-\beta_{i}\right| \leq\left(r+\frac{r_{\beta}^{\prime}}{3}\right)+\frac{r_{\beta}}{2} \\
& \leq\left(r_{2}+\frac{r_{\beta}^{\prime}}{3}\right)+\frac{r_{\beta}^{\prime}}{6} \leq 2 \frac{r_{\beta}^{\prime}}{3},
\end{aligned}
$$

since $r_{2} \leq r_{\beta} / 2$. It follows that $D_{k}^{\prime} \subset \Delta_{\beta}^{\prime}$ and the fill-in $D_{k}$ of $D_{k}^{\prime}$ is also contained in $\Delta_{\beta}^{\prime}$. Therefore $H\left(D\left(f^{n_{p}^{\prime}}(x), r\right)\right) \supset D\left(F^{n_{p}^{\prime}}(H(x)), r^{\prime}\right)$, and the degree of the restriction of $F^{n_{p}^{\prime}}$ to any connected component of $F^{-n_{p}^{\prime}}\left(D\left(F^{n_{k}+j}(H(x)), r^{\prime}\right)\right)$ is at most $\delta$ (cf. the definition of $r_{\beta}^{\prime}$ ).

This implies that $F$ is weakly hyperbolic. 
Proof of Theorem 2.1. We first assume that we are under the normalization fixing $(0,1, \infty)$. It follows from Proposition 2.3 that $\left(h_{t}\right)$ is an equicontinuous family, and that so is $\left(f_{t}\right)$. To prove the convergence of the deformation, it suffices to prove the uniqueness of the limits as $t \rightarrow 1$.

Therefore, we may now assume that there are sequences $\left(t_{n}\right)$ and $\left(s_{n}\right)$ tending to 1 such that $h_{t_{n}} \rightrightarrows H_{1}, h_{s_{n}} \rightrightarrows H_{2}, f_{t_{n}} \rightrightarrows F_{1}$, and $f_{s_{n}} \rightrightarrows F_{2}$. As $H_{1,2}$ have the same fiber systems, there is a homeomorphism $\phi$ making the following diagram commute:

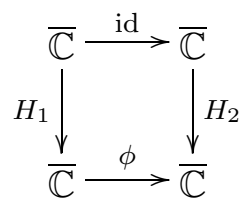

We claim that $H_{i}\left(J_{f} \cup \mathcal{R}\right)=J_{F_{i}}$, for the left hand set is $F_{i}$-fully invariant, has no isolated points, and periodic points are dense inside. This implies that $\phi$ is a topological conjugacy from $F_{1}$ to $F_{2}$. Moreover $\phi$ restricted to the Fatou set $\mathcal{F}$ of $F_{1}$ is equal to $H_{2} \circ H_{1}^{-1}$. But due to our technical assumption on the complex structure $\sigma_{t}$, on any compact set of $H_{1}^{-1}(\mathcal{F})$ the two maps $H_{1}$ and $\mathrm{H}_{2}$ integrate the same complex structure (this is still true even without the technical assumption, but the proof is more delicate). By local uniqueness the map $\phi$ is conformal on $\mathcal{F}$. The key point here is to apply a rigidity result of Haïssinsky, restated in Theorem 2.12 below, to conclude that in this case $\phi$ is globally conformal. But it fixes $0,1, \infty$ due to normalization. So $\phi=\mathrm{id}$. This gives the convergence of $\left(h_{t}\right)$, with the same fiber system, i.e. properties (I) and (II). The other properties follow.

THEOREM 2.12. Let $f$ be a weakly hyperbolic rational map which is not a Lattès example. Assume that $\varphi$ is a homeomorphism of $\overline{\mathbb{C}}$, conformal on $\overline{\mathbb{C}} \backslash J_{f}$, such that $\varphi \circ f \circ \varphi^{-1}$ is again a rational map. Then $\varphi$ is a Möbius transformation.

See [11] for a proof.

2.5. Equicontinuity of $\left(h_{t}\right)$ at $z_{0} \in \mathcal{R} \cup\{$ preparabolic points (Step 3). The idea is to compare deformations of local dynamics with that of model parabolic dynamics.

For each $\nu \in \mathbb{N}$, we define the model parabolic maps $g_{\nu}(z)$ and the standard projection $\pi_{\nu}$ to be:

$$
\begin{aligned}
& g_{1}(z)=g(z)=\frac{z}{1-z}, \\
& g_{\nu}(z)=\left(\frac{g\left(\nu z^{\nu}\right)}{\nu}\right)^{1 / \nu}=z\left(\frac{1}{1-\nu z^{\nu}}\right)^{1 / \nu}=z\left(1+z^{\nu}+\cdots\right), \quad \pi_{\nu}(z)=\nu z^{\nu} .
\end{aligned}
$$


The map $g_{\nu}$ maps univalently $\mathbb{C} \backslash \pi_{\nu}^{-1}\left(\left[1, \infty[)\right.\right.$ onto $\mathbb{C} \backslash \pi_{\nu}^{-1}(]-\infty,-1[)$, and has a parabolic fixed point at 0 with $\nu$ attracting petals. Note that $\pi(z)=-1 / z$ conjugates $g(z)$ to the standard translation $T: z \mapsto z+1$. Therefore $\pi \pi_{\nu}$ is the Fatou coordinate for $g_{\nu}$.

A left sepal (resp. right sepal) for $g_{\nu}$ is an invariant region corresponding to an upper (resp. lower) half-plane $H$ in the $\pi \circ \pi_{\nu}(z)$ coordinates with $T H=H$. In case the boundary of $H$ is a straight line and $\nu=1$, the corresponding sepal is simply a round disk centered in $i \mathbb{R}$ tangent to 0 .

One defines similarly attracting (resp. repelling) petals corresponding to right (resp. left) half-planes $L$ with $T L \subset L$ (resp. $T^{-1} L \subset L$ ), and then attracting and repelling invariant sectors corresponding to right and left half-strips. A flower neighborhood corresponds to the union of a left and a right sepal, together with an attracting and a repelling petal.

When the boundaries of these regions are horizontal or vertical straight lines (in the $\pi \pi_{\nu}$ coordinate), we call them straight sepals, petals, etc.

Lemma 2.13. Given any parabolic germ $F$ with $\nu$ attracting fixed petals at 0 , there is a neighborhood $V$ of 0 for $F$, a straight flower neighborhood $U$ of 0 for $g_{\nu}$, and a qc-conjugacy $\varphi$ from $\left(g_{\nu}(z), U\right)$ to $(F(z), V)$. Moreover $\varphi$ can be chosen to be conformal on the sepals and on the repelling petals.

Proof. We shall construct $\chi=\varphi^{-1}$.

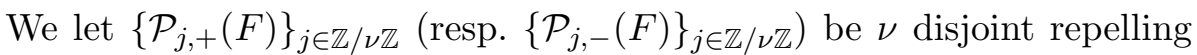
(resp. attracting) petals of $F$ numbered in cyclic order such that $\mathcal{P}_{j,+}(F)$ lies between $\mathcal{P}_{j,-}(F)$, and $\mathcal{P}_{j+1,-}(F)$. Let $\mathcal{S}_{l, j}(F)$ be a sepal which intersects $\mathcal{P}_{j,+}(F)$ and $\mathcal{P}_{j+1,-}(F)$, and $\mathcal{S}_{r, j}(F)$ be a sepal which intersects $\mathcal{P}_{j,+}(F)$ and $\mathcal{P}_{j,-}(F)$. We let $v_{j}(F)$ be the repelling axis of $\mathcal{P}_{j,+}(F)$. Finally, we let $\Phi_{j, \pm}: \mathcal{P}_{j, \pm}(F) \rightarrow \mathbb{C}$ be Fatou coordinates.

We define $\left\{v_{j}\left(g_{\nu}\right)\right\}_{j \in \mathbb{Z} / \nu \mathbb{Z}}$ to be the $\nu$ repelling axes of $g_{\nu}$ numbered in cyclic order.

Let $\chi: \mathcal{P}_{j,+}(F) \rightarrow \mathbb{C}$ be defined by $\chi(z)=\left(\pi \pi_{\nu}\right)^{-1} \circ \Phi_{j,+}(z)$ with the inverse branch of $\left(\pi \pi_{\nu}\right)$ chosen so that, for all $j \in \mathbb{Z} / \nu \mathbb{Z}, \chi$ maps $v_{j}(F)$ to $v_{j}\left(g_{\nu}\right)$. Restricting $\mathcal{P}_{j,+}(F)$ if necessary, we may assume that its image is contained in $\mathbb{D}$. It follows that

$$
\chi \circ F=g_{\nu} \circ \chi
$$

on $F^{-1}\left(\bigcup_{j} \mathcal{P}_{j,+}(F)\right)$.

Therefore, we may use this functional equation to extend $\chi$ univalently to the sepals, shortening them if necessary. It remains to extend $\chi$ quasiconformally to invariant attracting sectors.

For each $j \in \mathbb{Z} / \nu \mathbb{Z}$, we let $\mathcal{P}_{j}^{\prime} \subset \mathcal{P}_{j,-}$ be disjoint attracting invariant sectors which cover the set on which $\chi$ is not defined. On the attracting 
quotient cylinder $\mathbb{C} / \mathbb{Z}, \mathcal{P}_{j}^{\prime}$ is an annulus. We assume that its modulus is finite and that its boundary is composed of two disjoint horizontal curves.

It follows from the uniqueness of Fatou coordinates up to an additive constant that the expressions of $\chi$ on those two curves differ by a constant. Therefore, a quasiconformal extension of $\chi$ exists which conjugates the dynamics. The map $\varphi=\chi^{-1}$ satisfies the requirement of the lemma.

Let us return to our rational map $f$. Let $\gamma=\gamma_{i}$ be one of the repelling

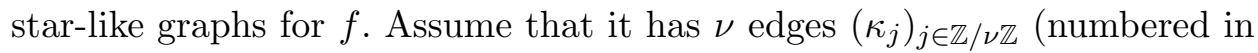
cyclic order). Denote by $q$ the (common) period of $\kappa_{j}$. Choose a smooth Jordan neighborhood $\Delta(\beta)$ of $\beta$ so that its boundary intersects (transversally) each $\kappa_{j}$ at only one point. A flower neighborhood of $\gamma$ is in the form $\Delta(\beta) \cup \bigcup_{j}\left(B^{\prime}\left(\kappa_{j}\right) \cup \Delta\left(\alpha\left(\kappa_{j}\right)\right)\right)$, that is, like a blue set.

LEMMA 2.14. There is a flower neighborhood $V$ of $\gamma$, a flower neighborhood $U$ of 0 for the model map $g_{\nu}$, a collection $R$ of closed sepals, one in each sepal of $U$, and a qc-conjugacy $\varphi$ from $\left(U \backslash R, g_{\nu}\right)$ to $\left(V \backslash \gamma, f^{q}\right)$, such that $\varphi$ maps conformally $\left(\operatorname{sepals}(U) \backslash R\right.$ onto $\bigcup_{j} B^{\prime}\left(\kappa_{j}\right) \backslash \gamma$, and $\varphi$ is conformal on a union $C$ of invariant repelling sectors with $\varphi(C) \supset$ $\left(J_{f} \cup \mathcal{G}\right) \cap\left(\Delta(\beta) \backslash \bigcup_{j} B^{\prime}\left(\kappa_{j}\right)\right)$.

Proof. We proceed as in the previous lemma and define the inverse map $\chi=\varphi^{-1}$. Let $V$ be a flower neighborhood of $f$ as above, and let $\widehat{\psi}: \Delta(\beta) \backslash$ $\{\beta\} \rightarrow \mathbb{C}$ be a multivalued, locally univalent map which satisfies $\widehat{\psi} \circ f^{q}(z)=$ $\widehat{\psi}(z)+1$.

1. For each $\kappa_{j}$, recall that $B^{\prime}\left(\kappa_{j}\right)$ is the invariant strip neighborhood chosen in the set up of simple pinching.

Let $\left\{\mathcal{P}_{j,+}\right\}_{j \in \mathbb{Z} / \nu \mathbb{Z}}$ be the connected components of $\Delta(\beta) \backslash \gamma$ numbered in cyclic order.

2. For each $j$, choose an invariant subsector with piecewise smooth boundary $\mathcal{P}_{j,+}^{\prime}$ such that

$$
\left(J_{f} \cup \mathcal{G}\right) \cap\left(\Delta(\beta) \backslash \bigcup_{i} B^{\prime}\left(\kappa_{i}\right)\right) \subset \mathcal{P}_{j,+}^{\prime} \subset \mathcal{P}_{j,+} \backslash \bigcup_{i} \overline{B^{\prime}}\left(\kappa_{i}\right)
$$

(we assume that the boundaries intersect just at $\beta$ and $\partial \Delta(\beta)$ ).

3. Around each repelling axis $v_{j}\left(g_{\nu}\right)$, choose a straight invariant sector neighborhood $C_{j}$ such that the quotient Riemann surfaces have the same moduli:

$$
\bmod C_{j} / g_{\nu}=\bmod \mathcal{P}_{j,+}^{\prime} / f^{q}
$$

Define $\chi:\left(\mathcal{P}_{j,+}^{\prime}, f^{q}\right) \rightarrow\left(C_{j}, g_{\nu}\right)$ to be a conformal conjugacy (adjusting the outer boundary of $C_{j}$ if necessary).

4. For each attracting axis of $g_{\nu}$, choose one left sepal $\mathcal{S}_{j, l}$ and one right sepal $\mathcal{S}_{j, r}$ disjoint from $\bigcup_{i} C_{i}$. 
5. Inside each sepal $\mathcal{S}_{j, l}$, define $R_{j, l}$ to be the uniquely determined subsepal so that

$$
\bmod \left(\mathcal{S}_{j, l} \backslash R_{j, l}\right) / g_{\nu}=\bmod B^{\prime l}\left(\kappa_{j}\right) / f^{q}
$$

(where $B^{\prime l}\left(\kappa_{j}\right)$ is the left component of $\left.B^{\prime}\left(\kappa_{j}\right) \backslash \kappa_{j}\right)$.

Define $\chi:\left(B^{\prime l}\left(\kappa_{j}\right), f^{q}\right) \rightarrow\left(\mathcal{S}_{j, l} \backslash R_{j, l}, g_{\nu}\right)$ to be a conformal conjugacy.

Do the same for each right sepal.

6. Extend $\chi$ quasiconformally to the domains $\Delta\left(\alpha_{j}\right) \backslash B^{\prime}\left(\kappa_{j}\right)$ and $\Delta(\beta) \backslash$ $\bigcup_{i}\left(B^{\prime}\left(\kappa_{i}\right) \cup \mathcal{P}_{i,+}^{\prime}\right)$. In both cases, the boundaries are periodic half-strips in the log-linearizing coordinates for $\Delta\left(\alpha_{j}\right) \backslash B^{\prime}\left(\kappa_{j}\right)$ and in $\widehat{\psi}$-coordinates for $\Delta(\beta) \backslash$ $\bigcup_{i}\left(B^{\prime}\left(\kappa_{i}\right) \cup \mathcal{P}_{i,+}^{\prime}\right)$, and the restriction of $\chi$ to the boundary commutes with translation by 1 . This ensures the existence of a quasiconformal extension which conjugates the dynamics. The map $\varphi=\chi^{-1}$ satisfies the requirements of the lemma.

Proposition 2.15 (equicontinuity of $\left(h_{t}\right)$ at $z_{0} \in \mathcal{R}$ ). Fix $z_{0} \in \gamma$. For any $\varepsilon>0$, there is $\delta>0$ such that for any $\left|z-z_{0}\right|<\delta$ and any $t$, we have $\left|h_{t}(z)-h_{t}\left(z_{0}\right)\right|<\varepsilon$.

Proof. For simplicity we will only treat the case that $\gamma$ has a unique edge $\kappa$.

Let $\varphi, U, R$ be as in the above lemma. Note that $\pi=\pi^{-1}$. Denote by $B^{l}$ the left sepal of $U$. There is a translation $T_{\sigma}$ by a pure imaginary constant, mapping $\pi\left(B^{l}\right)$ to $\left\{y>L_{b}\right\}$ and $\pi\left(B^{l} \backslash R\right)$ to $\left\{L_{b}<y<L_{r}\right\}$. Define $P_{t}(z)$ on $B^{l}$ to be $\pi T_{\sigma}^{-1} \widetilde{P}_{t} T_{\sigma} \pi(z)$. Define $P_{t}(z)$ on the right sepal $B^{r}$ similarly. It extends by identity to the remaining part of $U$.

Set $\mu_{t}=\varphi^{*} \sigma_{t}=\mu_{t}^{b} \wedge \mu_{t}^{c} \wedge \mu^{s}$, where we have decomposed $\mu_{t}$ into three structures with disjoint supports $B\left(=B^{l} \cup B^{r}\right), C$ and $S$ respectively. Note that $\mu_{t}^{s}=\mu^{s}$ is independent of $t$.

We will integrate them one by one: first $P_{t}$ integrates $\mu_{t}^{b}$, conjugates $g$ to $g$, and is the identity on $S \cup C$. The next step $C_{t}$ integrates $\mu_{t}^{c}$, and conjugates $g$ to a germ $X_{t}$. Finally, $S_{t}$ integrates $\left(C_{t}\right)_{*} \mu^{s}$, it is a $K$-qc map with $K$ independent of $t$, and conjugates $X_{t}$ to a parabolic germ $Y_{t}$.

We have

$$
\left.h_{t}\right|_{V}=\left.H^{-1} \circ S_{t} \circ C_{t} \circ P_{t} \circ \varphi^{-1}\right|_{V},
$$

where $H$ is a suitable univalent map.

Let us end up the proof assuming the following lemma.

Lemma 2.16. For any $M>0$, there are $t_{0}<1$ and a closed neighborhood $\widehat{E}$ of $\gamma$ such that for any $t>t_{0}, \bmod h_{t}(V \backslash \widehat{E})>M$.

Due to the normalization, Lemma 2.16 implies that

Given any $\varepsilon>0$, there are $t_{0}<1$ and a closed neighborhood $\widehat{E}$ of $\gamma$ such that for any $t>t_{0}$, diam $h_{t}(\widehat{E})<\varepsilon$. 


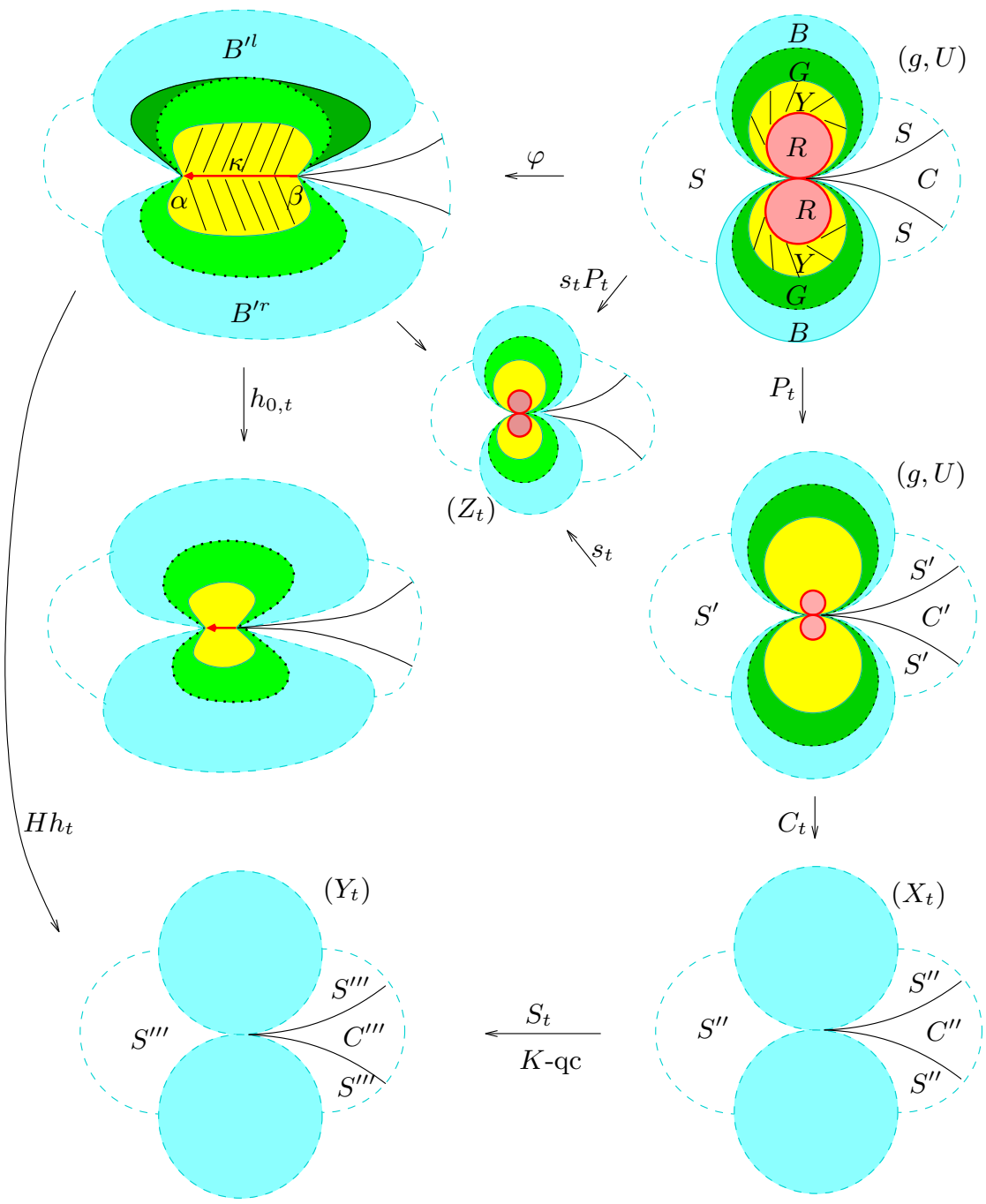

Fig. 4. Relations with various models

This, together with the continuity of $h_{t}(z)$ on $(t, z) \in\left[0, t_{0}\right] \times \overline{\mathbb{C}}$, gives the proposition.

In order to prove Lemma 2.16, we first study the properties of $P_{t}$ and $C_{t}$ :

Property of $P_{t}$ : For any $r>0$, there are $t_{0}<1$ and $E$ a full continuum neighborhood of $R$ such that for any $t \geq t_{0}, P_{t}(E) \subset \bar{D}_{r}$.

This is due to the continuity of $P_{t}(z)$ on $(t, z)$ with $P_{1}(R)=\{0\}$.

PRoperty of $C_{t}$ : For some sequence $r_{k} \searrow 0, \bmod C_{t}\left(U \backslash \bar{D}_{r_{k}}\right) \rightarrow \infty$ as $r_{k} \rightarrow 0$ uniformly in $t \in[0,1[$. 
Proof. Note that $\pi(C)$ is a left half-strip of the form $\left\{x \leq a^{\prime}<0\right.$, $|y| \leq b\}$. Set $\mathcal{G}^{\prime}=\pi(\mathcal{G} \cap C)$. Denote by $G^{\prime}$ a general $\mathcal{G}^{\prime}$-component. For $a \in \mathbb{R}, a<a^{\prime}$, define

$$
\Sigma_{a}=\{x=a,|y| \leq b\} \cup \underset{G^{\prime} \cap\{x \equiv a\} \neq \emptyset}{\bigcup} \overline{G^{\prime}} .
$$

All disks $D\left(r_{i}\right)$ below are centered at 0 . Choose one by one $R_{0}^{-}>0, x_{0}<0$, $R_{0}^{+}>0$ and $N \in \mathbb{N}$ such that

$\partial D\left(R_{0}^{-}\right) \subset \pi(U), \Sigma_{x_{0}} \cap \bar{D}\left(R_{0}^{-}\right)=\emptyset, \Sigma_{x_{0}} \subset \bar{D}\left(R_{0}^{+}\right), T^{-N} \Sigma_{x_{0}} \cap \bar{D}\left(R_{0}^{+}\right)=\emptyset$.

Fix $k \in \mathbb{N}$. Set $x_{k}=x_{0}-k N$. Let $R_{k}^{-}$be the radius of the largest disk not intersecting the interior of $\Sigma_{x_{k}}$. Define $R_{k}^{+}$such that $-R_{k}^{+}-x_{k}=-R_{0}^{+}-x_{0}$ $=: C_{0}$. Set $r_{k}=1 / R_{k}^{+}$and let $Q$ be the rectangle $\left\{\log R_{0}^{-}<x<\log R_{k}^{+}\right.$, $|y| \leq \pi\}$. Then

$$
\bmod C_{t}\left(U \backslash \bar{D}_{r_{k}}\right) \geq \bmod C_{t}\left(\pi\left(\left\{R_{0}^{-}<|z|<R_{k}^{+}\right\}\right)\right)=\bmod C_{t} \pi\left(-e^{Q}\right) .
$$

Define now $\varrho$ to be the density of the Euclidean metric on $Q$ minus the yellow set (more precisely on $Q \backslash \log \left(-\pi\left(\varphi^{-1}(\mathcal{Y} \cap \varphi(C))\right)\right)$ ), and to be zero elsewhere. Let $\Gamma$ be the set of $\operatorname{arcs}$ in $Q$ connecting the two vertical segments. Then

$$
\bmod C_{t}\left(U \backslash \bar{D}_{r_{k}}\right) \geq \frac{L_{\varrho}(\Gamma)^{2}}{\operatorname{Area}_{\varrho}(Q)} \stackrel{*}{\geq} \frac{\left(c\left(\log R_{k}^{-}-\log R_{0}^{+}\right)\right)^{2}}{2 \pi\left(\log R_{k}^{+}-\log R_{0}^{-}\right)} \rightarrow \infty
$$

as $k \rightarrow \infty$, where $*$ is due to the following two facts:

1. Each arc in $\Gamma$ contains a subarc with end points outside $-\log \mathcal{G}^{\prime}$, of Euclidean length at least $\log R_{k}^{-}-\log R_{0}^{+}$, due to the construction of $R_{k}^{ \pm}$.

2. There is a uniform Koebe space around each green component in $Q$ so that the estimates in the Key Lemma still hold, probably with a different constant.

Now the limit follows from $R_{k}^{+}-R_{k}^{-} \leq R_{k}^{+}-\left(-x_{k}\right)+\operatorname{diam} \Sigma_{x_{k}}=C_{0}+$ $\operatorname{diam} \Sigma_{x_{0}}$ as $T_{k N} \Sigma_{x_{k}}=\Sigma_{x_{0}}$. This ends the proof of Property of $C_{t}$.

Proof of Lemma 2.16. For any $M>0$, there exists $r>0$ such that $\bmod \left(C_{t}\left(U \backslash \bar{D}_{r}\right)\right) \geq K M$ for any $t$; also there are $t_{0}, E$ such that $P_{t}(E) \subset \bar{D}_{r}$ for any $t>t_{0}$. Set $\widehat{E}=\varphi(E)$. Then for any $t>t_{0}$, $\bmod h_{t}(V \backslash \widehat{E})=\bmod H h_{t}(V \backslash \widehat{E})=\bmod S_{t} C_{t} P_{t}(U \backslash E)$ $\stackrel{S_{t} \text { is } K \text {-qc }}{\geq} \frac{1}{K} \bmod C_{t} P_{t}(U \backslash E) \stackrel{\text { Grötzsch }}{\geq} \frac{1}{K} \bmod C_{t}\left(U \backslash \bar{D}_{r}\right) \geq \frac{1}{K} K M=M$.

There are two variants of the above proof, one uses only the properties of $P_{t}$ (which is used in Cui's original manuscript), and the other only the properties of $C_{t}$. 


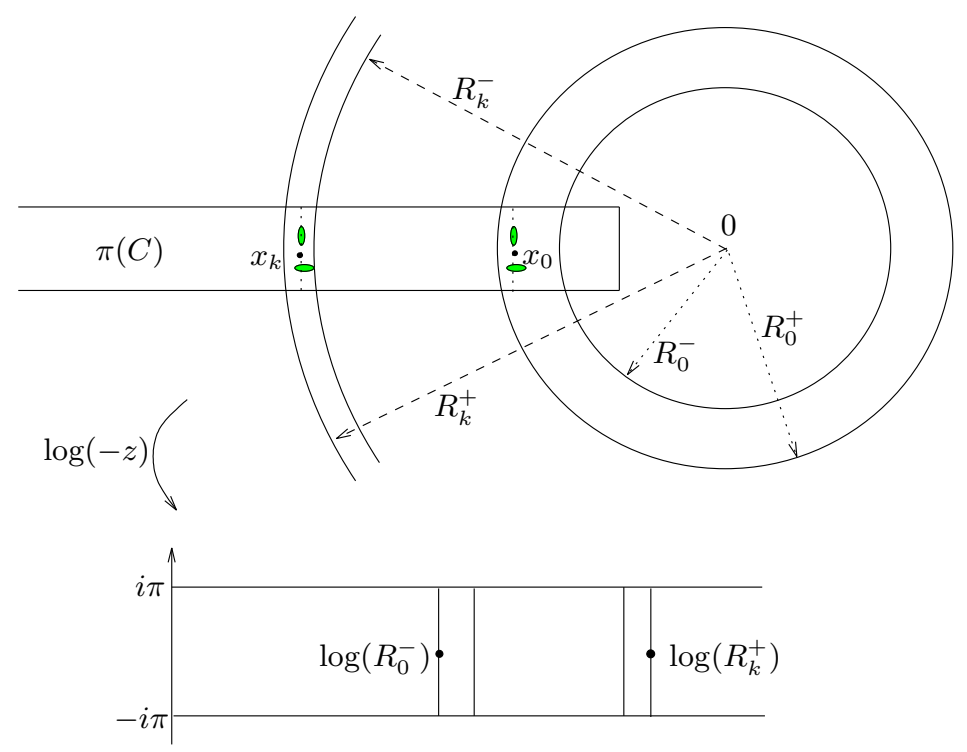

Fig. 5. Estimates in the log-coordinate

Denote by $h_{0, t}: V \rightarrow \overline{\mathbb{C}}$ the map that integrates the complex structure $\mu_{t}$ that is $\sigma_{t}$ on $B^{\prime}(\kappa)$ and $\sigma_{0}$ elsewhere, normalized so that $h_{0, t}(0)=0$ and $h_{0, t}(z)=z+O(1)$ at $\infty$.

Now $\left(P_{t} \circ \varphi^{-1}\right)_{*}\left(\mu_{t}\right)$ is $g$-invariant with dilatation $K$ on $S$, and conformal elsewhere. Denote by $s_{t}$ the integrating map normalized as $h_{0, t}$. Then $s_{t}$ conjugates $g$ to a parabolic germ $Z_{t}$.

Then the argument above proves that $h_{0, t}$ is equicontinuous at $\gamma$ and $h_{0, t}(\gamma) \rightarrow\{0\}$ as $t \rightarrow 1$. (As this does not rely on the estimates in the Key Lemma, it works for nonsimple pinching as well, see [4].)

The other variant is the following:

Lemma 2.17. Let $F$ be a parabolic germ with $r$ petals, defined and univalent on a neighborhood $V$ of 0 . Assume that $\mathcal{Y} \subset \mathcal{G} \subset V$ satisfies:

(a) $\mathcal{G}$ is open, and $\bar{G}_{i} \rightarrow\{$ point $\}$ for any convergent sequence of distinct $\mathcal{G}$-components $G_{i}$,

(b) $F^{-1}(\mathcal{G}) \subset \mathcal{G}$,

(c) the Key Lemma holds for paths in $V$.

Then given any family of $F$-invariant structures $\sigma_{t}$ with support contained in $\overline{\mathcal{Y}}$, the integrating map $H_{t}$ (normalized as above) is equicontinuous at 0.

For this we use $\varphi$ as in Lemma 2.13, and then $C_{t}$ and $S_{t}$ as above.

Applying this lemma to $f^{l}$ near an $l$-periodic parabolic point, we get the equicontinuity of $h_{t}$ at these points. 
Finally, in order to prove equicontinuity of $h_{t}$ on backward components of $\gamma_{i}$ or at preparabolic points, we use the same technique as the one to find $c_{G}$ for preperiodic $G$. The details are omitted.

\section{MATINGS OF POLYNOMIALS}

3.1. Definition, existence and unicity. There are many equivalent ways to define matings of polynomials. We have chosen here the one presented by Milnor [18]:

Definition (the sphere). Following the terminology of Milnor, we consider $\mathbb{S}^{2}$ as the subspace of $\mathbb{C} \times \mathbb{R}$ defined by $\mathbb{S}^{2}=H_{+} \cup H_{-} \cup\{(z, 0) \in$ $\mathbb{C} \times \mathbb{R}:|z|=1\}$, where $H_{+}=\left\{(z, r) \in \mathbb{C} \times \mathbb{R}:|z|^{2}+r^{2}=1, r>0\right\}$ and $H_{-}=\left\{(z, r) \in \mathbb{C} \times \mathbb{R}:|z|^{2}+r^{2}=1, r<0\right\}$. Let $\tau_{ \pm}: \mathbb{C} \rightarrow H_{ \pm}$be the gnomonic projections defined by

$$
\tau_{+}(z)=(z, 1) / \sqrt{|z|^{2}+1}, \quad \tau_{-}(z)=(\bar{z},-1) / \sqrt{|z|^{2}+1} .
$$

The hemispheres $H_{ \pm}$are equipped with conformal structures by $\tau_{ \pm}$.

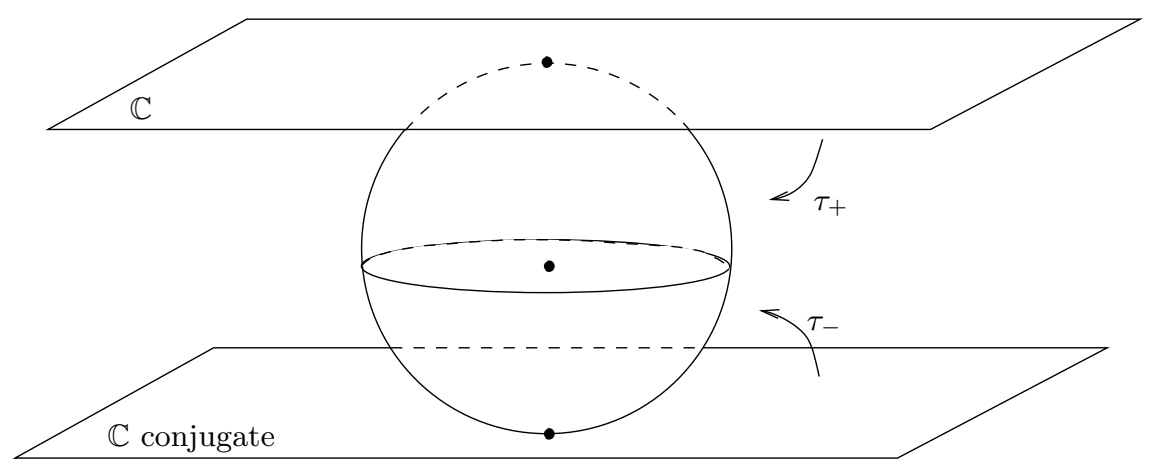

Fig. 6. Matings and gnomonic projections

Definition (topological mating). Let $F, G: \overline{\mathbb{C}} \rightarrow \overline{\mathbb{C}}$ be two degree $d$ ramified coverings. Here we allow $d$ to be 1 . Assume that $\infty$ is totally invariant for both $F$ and $G$, and $F$ and $G$ are holomorphic in a neighborhood of $\infty$ with local expansion $z^{d}(1+O(1 / z)$ ) (i.e. they have the same leading term in their local expansion at $\infty)$. We define the topological mating $F \Perp G$ : $\mathbb{S}^{2} \rightarrow \mathbb{S}^{2}$ to be the unique extension onto $\mathbb{S}^{2}$ of $\left.\tau_{+} \circ F \circ \tau_{+}^{-1}\right|_{H_{+}}$and $\tau_{-} \circ G \circ$ $\left.\tau_{-}^{-1}\right|_{H_{-}}$. By abuse of notation we will not distinguish $F$ and $\left.\tau_{+} \circ F \circ \tau_{+}^{-1}\right|_{H_{+}}$ (resp. $G$ and $\left.\tau_{-} \circ G \circ \tau_{-}^{-1}\right|_{H_{-}}$). An easy calculation shows that the map $F \Perp G$ is a well defined degree $d$ branched covering. In particular, if $d=1$ then we get a homeomorphism of the sphere. 
Assume now that $f$ and $g$ are two monic geometrically finite polynomials of degree $d \geq 2$ with connected Julia sets. The sphere $\mathbb{S}^{2}$ on which $f \Perp g$ is defined is equipped with the ray equivalence relation, which is defined to be the smallest equivalence relation generated by $x \sim y$ if $x, y$ belong to the closure in $\mathbb{S}^{2}$ of an external ray of $f$ or an external ray of $g$. The map $f \Perp g$ preserves this relation.

Definition. We say that $(f, g, q, R)$ is a marked mating if $f, g$ are monic polynomials of degree $d, R$ is a degree $d$ rational map and $q: \mathbb{S}^{2} \rightarrow \overline{\mathbb{C}}$ is a continuous map such that

(1) (semi-conjugacy) $q \circ(f \Perp g)=R \circ q$;

(2) (identification) $q(x)=q(y)$ if and only if $x$ and $y$ are ray-equivalent;

(3) (maximal conformality) $q$ is conformal in $\operatorname{int}\left(K_{f}\right) \cup \operatorname{int}\left(K_{g}\right), q\left(\operatorname{int}\left(K_{f}\right)\right.$ $\left.\cup \operatorname{int}\left(K_{g}\right)\right)=\overline{\mathbb{C}} \backslash J_{R}$ and $q^{-1}\left(\overline{\mathbb{C}} \backslash J_{R}\right)=\operatorname{int}\left(K_{f}\right) \cup \operatorname{int}\left(K_{g}\right)$.

We will say that two monic polynomials $f$ and $g$ are matable if there exist a continuous map $q$ and a rational map $R$ such that $(f, g, q, R)$ is a marked mating.

Unicity of $R$ and $q$. Pilgrim provided examples of nonunicity of the conformal conjugacy class of $R$ in a marked mating $(f, g, q, R)$ in case $R$ is a Lattès example. For details, see [18, Appendix B.9]. However, we have:

Proposition 3.1. Let $(f, g, q, R)$ be a marked mating. If $f, g, R$ are weakly hyperbolic and $R$ is not a Lattès example, then $R$ is unique up to conformal conjugacy, and for a given $R$, the map $q$ is unique up to postcomposition of an automorphism of $R$.

Proof. Consider two marked matings $(f, g, q, R)$ and $\left(f, g, q^{\prime}, R^{\prime}\right)$. By Lemma A.2 below, the map $q^{\prime} \circ q^{-1}$ is a homeomorphism conformal off the Julia set which conjugates $R$ to $R^{\prime}$. We conclude by the rigidity Theorem 2.12 .

We remark that it is not always easy to check that $R$ is weakly hyperbolic, knowing that $f$ and $g$ are weakly hyperbolic. But if $f$ and $g$ are geometrically finite, so is $R$. Moreover, without any normalization on $f$ and $g$, there are generally $d-1$ possible topological matings.

Existence. There are many known results regarding the existence and nonexistence of matings of postcritically finite polynomials, especially in degree 2 and 3. Interested readers can go to [18] and [24] for surveys of these results. The central tool is Thurston's theory for postcritically finite branched coverings. We mention here only the quadratic case: combining results of Levy, Rees, Shishikura and Tan, we have: 
Proposition 3.2. Two postcritically finite quadratic polynomials $f_{c}$ and $f_{c^{\prime}}$ are matable if and only if $c$ and $c^{\prime}$ do not belong to conjugate limbs of the Mandelbrot set.

See $[22,25]$ for more details. One of our tasks here is to extend this result to geometrically finite quadratic polynomials (Corollary C).

Existence of marked matings of some postcritically infinite polynomials can now follow from surgeries. We state this in the next proposition, which in particular provides us with an extension of Proposition 3.2 to hyperbolic polynomials with infinite critical orbits.

Proposition 3.3. Let $(f, g, q, R)$ be a marked mating of polynomials $f$ and $g$. Assume that $\widehat{f}$ (resp. $\widehat{g}$ ) is a quasi-regular map which coincides with $f$ (resp. $g$ ) on its basin of infinity, and that $\mu$ (resp. $\nu$ ) is an $\widehat{f}$-invariant (resp. $\widehat{g}$-invariant) Beltrami form supported on its filled-in Julia set. If we let $\varphi$ (resp. $\psi$ ) be a quasiconformal homeomorphism which integrates $\mu$ (resp. $\nu$ ) normalized at infinity to be tangent to the identity, then the polynomials $f_{1}=\varphi \circ \widehat{f} \circ \varphi^{-1}$ and $g_{1}=\psi \circ \widehat{g} \circ \psi^{-1}$ are matable.

Proof. Define $\varphi \Perp \psi: \mathbb{S}^{2} \rightarrow \mathbb{S}^{2}$ by

$$
\varphi \Perp \psi= \begin{cases}\tau_{+} \circ \varphi \circ \tau_{+}^{-1} & \text { on } H_{+}, \\ \mathrm{id} & \text { on the equator, } \\ \tau_{-} \circ \psi \circ \tau_{-}^{-1} & \text { on } H_{-} .\end{cases}
$$

The map $\varphi$ restricted to the basin of $\infty$ of $f$ realizes a conformal conjugacy from $f$ to $f_{1}$. The normalizations guarantee that $\varphi$ maps the $\theta$ external ray of $f$ to the ray of $f_{1}$ with the same angle. Therefore the map $\varphi \Perp \psi$ is a homeomorphism of $\mathbb{S}^{2}$, and a conjugacy from $\widehat{f} \Perp \widehat{g}$ to $f_{1} \Perp g_{1}$ which preserves the external rays of the same angle.

Now we define a " $q$-pushed forward" Beltrami form $\xi$ on the Riemann sphere as follows:

$$
\xi= \begin{cases}q_{*} \mu & \text { on } q\left(\operatorname{int} K_{f}\right), \\ q_{*} \nu & \text { on } q\left(\operatorname{int} K_{g}\right), \\ 0 \cdot d \bar{z} / d z & \text { elsewhere. }\end{cases}
$$

Since $q$ is conformal on $\operatorname{int}\left(K_{f}\right) \cup \operatorname{int}\left(K_{g}\right)$, the form $\xi$ is $R$-invariant. Therefore, the measurable Riemann mapping theorem provides us with a quasiconformal homeomorphism $\chi:(\overline{\mathbb{C}}, a, b, c) \rightarrow(\overline{\mathbb{C}}, a, b, c)$ which solves the Beltrami equation associated to $\xi$.

Set $R_{1}=\chi \circ R \circ \chi^{-1}$ and $q_{1}=\chi \circ q \circ(\varphi \Perp \psi)^{-1}: \mathbb{S}^{2} \rightarrow \overline{\mathbb{C}}$.

We can then check easily that $\left(f_{1}, g_{1}, q_{1}, R_{1}\right)$ is a marked mating. 
Remark. The proof of Theorem D will follow the same lines as Proposition 3.3, but instead of using the measurable Riemann mapping theorem, we shall use a generalization due to David [5].

\subsection{Continuous paths of matings}

Definition. Let $\left(f_{t}\right)_{t \in T}$ and $\left(g_{t}\right)_{t \in T}$ be two continuous families of matable polynomials. Let us say that they define a continuous family of matings if there are continuous families $\left(q_{t}\right)_{t \in T}$ and $\left(R_{t}\right)_{t \in T}$ such that $\left(f_{t}, g_{t}, q_{t}, R_{t}\right)_{t \in T}$ defines a family of marked matings. If $T=[0,1)$, we say that a continuous path of matings $\left(f_{t}, g_{t}, q_{t}, R_{t}\right)_{t \in T}$ is convergent if there exist limits $f, g, q$ and $R$ of $f_{t}, g_{t}, q_{t}$ and $R_{t}$ as $t$ tends to 1 such that $(f, g, q, R)$ is a marked mating.

We first need a preliminary result which is a version with parameter of Proposition 3.3.

Let $\left(f_{0}, g_{0}, q_{0}, R_{0}\right)$ be a marked mating of polynomials with connected Julia sets.

Let $\left(\widehat{f}_{t}\right)_{t \in T}$ be a continuous family of quasi-regular maps, each coinciding with $f_{0}$ on the basin of $\infty$ and mapping the interior of $K_{f_{0}}$ onto itself.

Let $\mu_{t}$ be $\widehat{f}_{t}$-invariant Beltrami forms, continuous in $t \in T$, and with support contained in $K_{f_{0}}$. The measurable Riemann mapping theorem provides us with a continuous family of quasiconformal maps $\varphi_{t}$, integrating $\mu_{t}$, and normalized to be tangent to the identity at infinity. Clearly $\varphi_{t}$ is conformal on the basin of $\infty$ for $f_{0}$.

Define $f_{t}=\varphi_{t} \circ \widehat{f}_{t} \circ \varphi_{t}^{-1}$. They are again monic polynomials with connected Julia set, and depend continuously on $t$.

Assume that $\widehat{g}_{t}, \nu_{t}, \psi_{t}, g_{t}$ are similar deformations of $g_{0}$.

Proposition 3.4. Under the above assumptions the marked matings $\left(f_{t}, g_{t}, q_{t}, R_{t}\right)_{t \in T}$ exist and depend continuously on $t$. In particular, if $\left(f_{t}\right)_{t \in[0,1)}$ and $\left(g_{t}\right)_{t \in[0,1)}$ are simple pinching deformations of $f_{0}$ and $g_{0}$ respectively (in this case $\widehat{f}_{t} \equiv f_{0}$ and $\widehat{g}_{t} \equiv g_{0}$ ), then $\left(R_{t}\right)$ is a simple pinching deformation of $R_{0}$ and $\left(f_{t}, g_{t}, q_{t}, R_{t}\right)_{t \in[0,1)}$ is a continuous path of marked matings.

Proof. We repeat the proof of Proposition 3.3 adding the subscript $t$. So we define subsequently the maps $\varphi_{t} \Perp \psi_{t}$, the $q_{0}$-pushed forward forms $\xi_{t}$ and the quasi-regular dynamics $\widehat{R}_{t}$. Since $q_{0}$ is conformal on $\operatorname{int}\left(K_{f_{0}}\right) \cup \operatorname{int}\left(K_{g_{0}}\right)$, the forms $\xi_{t}$ are $\widehat{R}_{t}$-invariant and depend continuously on $t$. Therefore, the measurable Riemann mapping theorem provides us with a continuous family of quasiconformal homeomorphisms $\chi_{t}:(\overline{\mathbb{C}}, a, b, c) \rightarrow(\overline{\mathbb{C}}, a, b, c)$ which solves the Beltrami equation associated to $\xi_{t}$.

$$
\text { Set } R_{t}=\chi_{t} \circ \widehat{R}_{t} \circ \chi_{t}^{-1} \text { and } q_{t}=\chi_{t} \circ q_{0} \circ\left(\varphi_{t} \Perp \psi_{t}\right)^{-1}: \mathbb{S}^{2} \rightarrow \overline{\mathbb{C}} \text {. }
$$


We can then check easily that $\left(f_{t}, g_{t}, q_{t}, R_{t}\right)_{t \in T}$ are marked matings and are continuous with respect to $t \in T$.

If $\left(f_{t}\right)_{t}$ and $\left(g_{t}\right)_{t}$ are simple pinchings, we check that the $q$-pushed forward Beltrami forms define a simple pinching deformation of $R_{0}$.

Now we can turn to the limit of simple pinchings. Consider simple pinchings $\left(f_{t}, \varphi_{t}\right)$ and $\left(g_{t}, \psi_{t}\right)$. We apply Proposition 3.4 and wish to prove Theorem $\mathrm{B}$, which can be summed up by the following diagram:

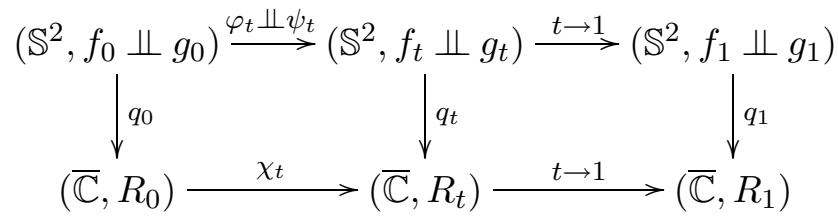

Proof of Theorem B. It follows from Proposition 3.4 that $\left(f_{t}, g_{t}, q_{t}, R_{t}\right)$ exists and is a marked mating for any $0 \leq t<1$, and that it depends continuously on $t$.

We can then apply Theorem 2.1 to all three deformations for $f_{0}, g_{0}$ and $R_{0}$ respectively and establish the convergences $f_{t} \rightrightarrows f_{1}, g_{t} \rightrightarrows g_{1}, \varphi_{t} \rightrightarrows \varphi_{1}$, $\psi_{t} \rightrightarrows \psi_{1}, R_{t} \rightrightarrows R_{1}$ and $\chi_{t} \rightrightarrows \chi_{1}$. One checks easily that $\varphi_{1} \Perp \psi_{1}$ is well defined and semi-conjugates $f_{0} \Perp g_{0}$ to $f_{1} \Perp g_{1}$. It remains to prove the uniform convergence of $q_{t}$ to a map $q_{1}$ as $t \rightarrow 1$, and that the limit 4-tuple $\left(f_{1}, g_{1}, q_{1}, R_{1}\right)$ is also a marked mating.

By our definition of pushed forward deformation, the map $q$ maps each equivalence class of $\varphi_{1} \Perp \psi_{1}$ (a red star in $K_{f_{0}}$ or in $K_{g_{0}}$ ) into an equivalence class of $\chi_{1}$.

Define now $q_{1}=\chi_{1} \circ q \circ\left(\varphi_{1} \Perp \psi_{1}\right)^{-1}$. By Lemma A.4, it has the following property:

(i) $q_{1}$ is well defined and continuous, $q_{t}$ converges uniformly to $q_{1}$ and $q_{1} \circ\left(\varphi_{1} \Perp \psi_{1}\right)=\chi_{1} \circ q$.

We have furthermore

(ii) $q_{1} \circ\left(f_{1} \Perp g_{1}\right)=R_{1} \circ q_{1}$.

Indeed, $q_{t} \circ\left(f_{t} \Perp g_{t}\right)=R_{t} \circ q_{t}$ and every map in the equation converges uniformly to the corresponding limit map.

(iii) $q_{1}(x)=q_{1}(y)$ iff $x$ and $y$ are ray-equivalent.

By (i) and Lemma A.3 we just need to show that $x \sim_{q} x^{\prime}$ implies $\varphi_{1} \Perp$ $\psi_{1}(x) \sim_{q_{1}} \varphi_{1} \Perp \psi_{1}\left(x^{\prime}\right)$. The map $\varphi_{1} \Perp \psi_{1}$, as the uniform limit of $\varphi_{t} \Perp \psi_{t}$, maps external rays of $f_{0} \Perp g_{0}$ bijectively onto external rays of $f_{1} \Perp g_{1}$ with the same angle. This is exactly what we wanted.

(iv) $q_{1}$ is univalent in $\operatorname{int}\left(K_{f_{1}}\right) \cup \operatorname{int}\left(K_{g_{1}}\right)$. 
Fix any open set $L$ compactly contained in $\operatorname{int}\left(K_{f_{1}}\right) \cup \operatorname{int}\left(K_{g_{1}}\right)$. For $t$ close to 1 the maps $q_{t}$ are univalent on $L$. So $q_{1}$ is either univalent or constant on $L$. The injectivity follows from (i) and (iii) above.

REMARK. The only assumptions that we have really used were the existence of the marked mating $\left(f_{0}, g_{0}, q_{0}, R_{0}\right)$ and the convergence of the three pinching deformations $\left(f_{t}, \varphi_{t}\right),\left(g_{t}, \psi_{t}\right)$ and $\left(R_{t}, \chi_{t}\right)$.

As a consequence, we get a positive answer to Milnor's original question (in a more general form):

Corollary 3.5. Let $f$ and $g$ be two quadratic hyperbolic polynomials in nonconjugate limbs of the Mandelbrot set. Then they are matable. Furthermore, assume that both maps have an attracting but non-superattracting periodic cycle. Then there exist a simple pinching path of $f$ and a simple pinching path of $g$. The mating of the two paths yields a continuous path of marked matings, and converges to a marked mating of parabolic polynomials. In the particular case that the multipliers of the attractors are real and positive, the pinching paths can be chosen so that the multipliers remain real and tend to 1 (this is stronger than radial convergence in the sense of McMullen).

Proof. In the hyperbolic component of $f$ there is a unique postcritically finite map $f_{0}$ (one can obtain this by surgery). Similarly for $g_{0}$. They do not belong to conjugate limbs of the Mandelbrot set. So Proposition 3.2 ensures that $f_{0}$ and $g_{0}$ are matable, and that $f$ and $g$ are matable as well.

To find a simple pinching combinatorics for $f$ and for $g$, we just need to lift a suitable simple closed curve in the quotient torus to the attractor. See for example [26].

Now we can apply Theorem B to conclude.

In the particular case with real positive multipliers for the attractors, we define an adapted pinching deformation. Let us first consider a conformal mapping which maps the Fatou component which contains the critical point to the unit disk. If it is properly normalized then it conjugates the first return map to

$$
B(z)=z \frac{z+\lambda}{1+\lambda z}
$$

on the unit disk with $\lambda \in(0,1)$. We will define the deformation for $B$ and then pull it back to our original map. We can check that the critical point of $B$ is on the segment $(-1,0)$, choose the pinching combinatorics to be the real segment $[0,1]$, and the Beltrami forms in the pinching deformations to be symmetric with respect to the real axis for $B$. These choices will guarantee that the deformed maps $f_{t}$ and $g_{t}$ continue to have a real positive multiplier for the attractor, and these multipliers tend to 1 at the limit of pinching. 
Proof of Corollary C. Let $c$ and $c^{\prime}$ be geometrically finite polynomials. If they belong to conjugate limbs of the Mandelbrot set, their respective $\alpha$-fixed point have opposite external angles and therefore $\mathbb{S}^{2} / \sim_{\text {ray }}$ is not a sphere and the marked mating cannot exist. See [25] for further details.

On the other hand, if they are not in conjugate limbs, then it is the same for $\mathcal{T}(c)$ and $\mathcal{T}\left(c^{\prime}\right)$, the center of the hyperbolic component having $c$, resp. $c^{\prime}$ as root. Therefore the marked mating of $f_{c}$ and $f_{c^{\prime}}$ exists by Corollary 3.5.

3.3. Theorems $\mathbf{D}$ and E. In this section, we prove Theorems D and E.

Postcritically finite polynomials associated to geometrically finite polynomials. Let $f$ be a monic and centered geometrically finite polynomial with connected Julia set of degree $d \geq 2$. Let us recall that there is a unique conformal map $B_{f}: \mathbb{C} \backslash \overline{\mathbb{D}} \rightarrow \mathbb{C} \backslash K_{f}$ which is tangent to the identity at infinity such that $B_{f}\left(z^{d}\right)=f \circ B_{f}(z)$. Since the Julia set is locally connected, this map extends continuously to the closure and induces the Carathéodory loop $\gamma_{f}: \mathbb{S}^{1} \rightarrow J_{f}$. We wish to associate with $f$ a canonical postcritically finite polynomial $\mathcal{T}(f)$. We will proceed in two steps. The first step follows from the statement proved in [10]:

Proposition 3.6. Let $f$ be a monic centered geometrically finite polynomial with connected Julia set. Then there exist continuous families of subhyperbolic polynomials $\left(f_{t}\right)_{0 \leq t<1}$ and of orientation preserving homeomorphisms $\left(h_{t}: J_{f} \rightarrow J_{f_{t}}\right)_{0 \leq t<1}$ such that $\left(f_{t}, h_{t}\right)$ tends to $(f, \mathrm{id})$ and such that $h_{t} \circ f=f_{t} \circ h_{t}$ on $J_{f}$. The maps $h_{t}$ are obtained as continuous extensions of the composition $B_{f_{t}} \circ B_{f}^{-1}$ of the Böttcher coordinates of $f$ and $f_{t}$, so $h_{t}=\gamma_{f_{t}} \circ \gamma_{f}^{-1}$ formally.

REMARKS. The construction of this perturbation is made in two steps: in the first step, we perturb $f$ in the space of polynomial-like mappings in order to destroy all parabolic points and to get a stable family; in the second step, the straightening theorem is used to get back to the polynomial world. The stability and the hand-made peturbation help showing that the mappings $h_{t}$ can be defined as continuous extensions of the Böttcher coordinates of $f$ and $f_{t}$. They have no particular regularity besides continuity in this statement. It follows that $K_{f}$ and $K_{f_{t}}$ are described by the same pinched disk model (cf. [6]) so that $h_{t}$ admits a homeomorphic extension to the plane which coincides with $B_{f_{t}} \circ B_{f}^{-1}$. It could be possible to prove that they admit $W_{\text {loc }}^{1,2}$ extensions to the plane, but this will not be needed in what follows (see however Corollary 3.10).

We would also like to emphasize that, at this point, the perturbation which is given by this proposition has nothing to do with pinching deforma- 
tions. But later on, we will use Proposition 3.6 to prove that we can actually choose it that way (see Proposition 3.11).

Given a monic and centered geometrically finite polynomial $f$ with connected Julia set, the proposition provides us with a (monic and centered) subhyperbolic polynomial map $\mathcal{S}(f)=f_{0}$ with a conjugacy $h: J_{f} \rightarrow J_{f_{0}}$. If $f$ is subhyperbolic, then we set $\mathcal{S}(f)=f$. By a simple surgery, we can then associate a canonical postcritically finite polynomial $\mathcal{T}(f)$ to $\mathcal{S}(f)$ (see for instance Theorem VI.5.1 of [3] or [15]).

We divide Theorem $\mathrm{D}$ into the next two propositions:

Proposition 3.7. Let $f, g$ be two monic and centered geometrically finite polynomials with connected Julia sets. If $\mathcal{T}(f)$ and $\mathcal{T}(g)$ are matable, then so are $f$ and $g$.

Proposition 3.8. Let $f, g$ be two monic and centered geometrically finite polynomials with connected Julia sets. If $f$ and $g$ are matable, then so are $\mathcal{T}(f)$ and $\mathcal{T}(g)$.

Our proof of Proposition 3.7 will rely on the following proposition [9].

Proposition 3.9 (parabolic surgery). Let $f$ be a subhyperbolic rational map with connected Julia set. Assume that $f$ has an attracting cycle $\alpha$ and a repelling cycle $\beta$ on the boundary of its immediate basin with period less than or equal to that of $\alpha$. Then there are another rational map $g$ and $a$ $\mu$-homeomorphism $\varphi$, locally quasiconformal in the Fatou set, univalent in the basin of $\infty$ and tangent to the identity at $\infty$, such that:

(i) $\varphi\left(J_{f}\right)=\varphi\left(J_{g}\right), \varphi(\beta)$ is parabolic and the immediate basin of $\alpha$ becomes that of $\varphi(\beta)$;

(ii) outside $\alpha$ 's immediate basin, $\varphi \circ f=g \circ \varphi$; in particular, $\varphi: J_{f} \rightarrow J_{g}$ is a homeomorphism which conjugates the dynamics.

The class of $\mu$-homeomorphisms is a generalization of quasiconformal maps [5]. It is stable under composition by quasiconformal maps and the associated Beltrami equation admits a unique normalized solution as in the measurable Riemann mapping theorem. For more details on $\mu$-homeomorphisms, we refer to [5]. Here also, the construction of $\varphi$ has nothing to do with pinchings, but this statement remains true for a much wider class than subhyperbolic maps.

COROLlARY 3.10. Let $f$ be a geometrically finite polynomial with connected Julia set. There is a $\mu$-homeomorphism $\varphi: \mathbb{C} \rightarrow \mathbb{C}$ such that $\varphi \circ$ $\mathcal{T}(f)=f \circ \varphi$ on $J_{\mathcal{T}(f)}$.

Proof. Since $\mathcal{T}(f)$ and $\mathcal{S}(f)$ are quasiconformally conjugate on their Julia sets, we may replace $\mathcal{T}(f)$ by $\mathcal{S}(f)$. Let us apply Proposition 3.9 to $\mathcal{S}(f)$ at points which used to be parabolic for $f$. One obtains a polynomial $F$ 
with a homeomorphism $h: J_{f} \rightarrow J_{F}$ such that $h \circ f=F \circ h$. This yields a correspondence between the Fatou components of $f$ and $F$ which has the property of matching the periodic points and components of the same nature together, and the restrictions of $f$ and $F$ to these domains have the same degree. It follows from McMullen's Propositions 6.7 and 6.8, and Theorem 6.1(d) from [15], that we may extend $h$ as a plane homeomorphism which will be quasiconformal off the Julia sets. The rigidity Theorem 2.12 then implies that it is globally quasiconformal so that $f$ and $F$ are quasiconformally conjugate. Since the class of $\mu$-homeomorphisms is preserved under the composition of quasiconformal maps, we have established the corollary.

We are now ready to prove Proposition 3.7.

Proof of Proposition 3.7. We proceed as in Proposition 3.3. Let us apply Corollary 3.10 to $f$ and $g$, and denote by $\varphi$ and $\psi$ the $\mu$-homeomorphisms that are given.

We denote by $(\mathcal{T}(f), \mathcal{T}(g), q, R)$ a marked mating. We let $\mu$ and $\nu$ be the Beltrami forms associated to $\varphi$ and $\psi$. We push them forward by $q$ on $\overline{\mathbb{C}}$ and denote the result by $\xi$. Define

$$
\left.H=q \circ\left(\varphi^{-1} \circ f \circ \varphi\right) \Perp\left(\psi^{-1} \circ g \circ \psi\right)\right) \circ q^{-1} .
$$

This map is well defined and we can check that it is ACL. Moreover, $\xi$ is $H$-invariant, and we may complete the proof analogously to the proof of Proposition 3.3 using David's generalization of the measurable Riemann mapping theorem (see [9] for further details).

Remark. We have used Proposition 3.9 because this strategy can be applied to a larger variety of matings for which we wish to create parabolic points. We could have used pinching techniques instead.

We propose an alternative proof of Corollary $\mathrm{C}$ which does not use pinching deformations.

Proof of Corollary $C$. Let $c$ and $c^{\prime}$ be geometrically finite polynomials. If they belong to conjugate limbs of the Mandelbrot set, we argue as in the first proof.

On the other hand, if they are not in conjugate limbs, then the same holds for $\mathcal{T}(c)$ and $\mathcal{T}\left(c^{\prime}\right)$, the center of the hyperbolic component having $c$, resp. $c^{\prime}$ as its root. Therefore the marked mating of $f_{c}$ and $f_{c^{\prime}}$ exists by Proposition 3.7.

It remains to prove Proposition 3.8 and Theorem E. The following will be used several times. 
Proposition 3.11. Let $f$ be a geometrically finite polynomial with connected Julia set. There exist a subhyperbolic map $f_{0}$ and a simple pinching deformation of $f_{0}$ which converges to $f$.

REMARK. Cui has proved this result for geometrically finite rational maps, regardless of whether the Julia set is connected or not [4]. But his proof is more involved since it relies on Thurston's characterization of rational maps.

Proof. We assume that $f$ is monic and centered. We denote by $A_{\text {par }}$ the union over all the parabolic points of their basin of attraction, and by $A_{\mathrm{par}}^{*}$ their immediate basins.

Proposition 3.6 enables us to define a first continuous family $\left(\widehat{f}_{t}\right)_{0 \leq t<1}$ of monic, centered, subhyperbolic polynomials with homeomorphims $h_{t}$ : $J_{f} \rightarrow J_{\widehat{f}_{t}}$ which conjugates the dynamics. Furthermore, for each $t \in[0,1)$, the map $h_{t} \circ h_{0}^{-1}: J_{\widehat{f}_{0}} \rightarrow J_{\widehat{f}_{t}}$ extends to a $K_{t}$-quasiconformal homeomorphism $\widehat{\varphi}_{t}$ where $K_{t}$ is a nondecreasing function of $t$ and $\widehat{\varphi}_{0}=$ id because they lie in a stable analytic family of polynomials (cf. Prop. 4.2 in [10]). These maps coincide with the composition of the Böttcher coordinates off the filled-in Julia sets.

Actually, we may assume that $\widehat{\varphi}_{t}$ is uniformly quasiconformal off the closure of the new attracting domains. To see this, it is enough to restrict ourselves to the periodic attracting cycles of $f$. Conjugating the first return maps $\widehat{f}_{t}^{k}$ to finite Blaschke products of the unit disk by Riemann maps, we see that we get a family of rational maps which are uniformly hyperbolic.

Let us note that this perturbation comes with a simple pinching combinatorics $\widehat{R}_{t}$ which link together all the new attracting and repelling points created by the desingularization of the parabolic points (cf. Prop. 2.1 in [10]).

Of course, there are no reasons why this deformation could be interpreted as a pinching deformation. The rest of the proof will be broken into three steps. They consist in "correcting" this path of polynomials to make it a pinching path. The first step will fix the right dynamics in the Fatou components which we wish to keep intact, the second step will study the limit of the simple pinching associated to the combinatorics coming from the above, and the third will provide us with the sought pinching deformation.

STEP 1. If $f$ has no attracting point, then we define $\widehat{F}_{t}=\widehat{f}_{t}$, and $\psi=\mathrm{id}$, and we may directly proceed to Step 2 . Otherwise, let $\alpha \in \mathbb{C}$ be an attracting periodic point of $f$ of period $k$. Let $U$ be the Fatou component containing $\alpha$. For all $t$, we denote by $\widehat{\alpha}_{t}$ the perturbation of $\alpha$ and $\widehat{U}_{t}$ the Fatou component bounded by $h_{t}(\partial U)$.

Since the Julia sets are locally connected, both components $U$ and $\widehat{U}_{0}$ are Jordan domains. Furthermore, the restrictions of $f^{k}$ and $\widehat{f}^{k}$ to these 
domains have the same degree and contain an attracting point by continuity. Therefore, Proposition 6.7 in [15] implies that there is a quasiconformal homeomorphism $\widehat{h}_{0}: U \rightarrow \widehat{U}_{0}$ which coincides with $h_{0}$ on the boundary. We proceed similarly for all bounded Fatou components disjoint from $A_{\text {par }}$. We note that the quasiconformal distortion can be chosen to be uniformly bounded. For each $t$, we may define $h_{t}=\widehat{\varphi}_{t} \circ h_{0}$.

Let us define a new family of maps $g_{t}$ by setting $g_{t}=h_{t} \circ f \circ h_{t}^{-1}$ where $h_{t}^{-1}$ is defined, and $g_{t}=\widehat{f}_{t}$ elsewhere. Each map $g_{t}$ is continuous. Since $g_{t}$ is quasi-regular on the complement of where it coincides with $\widehat{f}_{t}$, Rickman's removability theorem implies that $g_{t}$ is a well defined quasi-regular map (see [21] or Lemma I.2 in [7]). Furthermore, we may define a $g_{t}$-invariant ellipse field $E_{t}$ by setting $E_{t}=\left(h_{t}\right)_{*} \mathbb{S}^{1}$ on the domain of $h_{t}^{-1}$ and $E_{t}=\mathbb{S}^{1}$ elsewhere, where $\mathbb{S}^{1}$ denotes the conformal structure induced by the standard complex structure. Thus, the measurable Riemann mapping theorem provides us with a quasiconformal homeomorphism $\psi_{t}$ and a monic centered polynomial $\widehat{F}_{t}=\psi_{t} \circ g_{t} \circ \psi_{t}^{-1}$. It follows from the construction that $\left(\psi_{t}\right)$ is a normalized family of uniformly quasiconformal homeomorphisms, so that there is a quasiconformal map $\psi$ which is the limit of a convergent sequence $\left(\psi_{t_{n}}\right)_{n}$ with $t_{n} \rightarrow 1$.

Therefore, one also gets a limit $\widehat{F}$ of $\left(\widehat{F}_{t_{n}}\right)$. The maps $\psi_{t} \circ h_{t}$ are conformal on $\mathbb{C} \backslash \bar{A}_{\text {par }}$, so we get a conformal conjugacy between $f$ and $\widehat{F}$ on this set. Furthermore, for any $z \in J_{f}, \psi_{t_{n}} \circ h_{t_{n}}(z)$ tends to $\psi(z)$. On the other hand, on any compact subset of $A_{\text {par }}, g_{t}=\widehat{f}_{t}$ for $t$ close enough to 1 , so that $\widehat{F}=\psi \circ f \circ \psi^{-1}$. Therefore $f$ and $\widehat{F}$ are conjugate by a homeomorphism which is conformal off $J_{f}$. It follows from Theorem 2.12 and the normalization that $\widehat{F}=f$. Furthermore, the whole path $\left(\widehat{F}_{t}\right)_{t}$ converges to $f$ since it has only one accumulation point.

SteP 2. We may define a simple pinching deformation $\left(F_{t}, \phi_{t}\right)$ of $F_{0}=$ $\widehat{F}_{0}$ supported by $\psi_{0}\left(\widehat{R}_{0}\right)$ with $\phi_{t}(z)=z+o(1)$ at infinity. We let $\mathcal{Y}$ be the yellow set for $F_{0}$. It follows from Theorem 2.1 that this deformation converges to some $(F, \phi)$. The map $H=\phi \circ \psi_{0} \circ h_{0}$ defined off $A_{\text {par }}$ defines a conjugacy between $f$ and $F$ which is conformal off $\bar{A}_{\text {par }}$.

We end this step by proving that $H$ admits a quasiconformal extension to $\mathbb{C}$ which will extend the conjugacy. We will need more knowledge on $\widehat{f}_{t}$ to proceed. We will refer to [10] when this is needed. The conjugacy between the parabolic basins will be first defined on the level of Fatou coordinates and then lifted to the dynamical planes.

Let $\beta_{f}$ be a parabolic point for $f$, and denote by $A\left(\beta_{f}\right)$ its basin of attraction and by $\Phi_{f}: A(\beta) \rightarrow \mathbb{C}$ a Fatou coordinate that will be normalized later on. We define $\beta_{F}=\psi\left(\beta_{f}\right)$ ( $\psi$ has been defined in Step 1), $A\left(\beta_{F}\right)$ and $\Phi_{F}$ similarly. 
Let $c_{1}, \ldots, c_{\nu}$ be the critical points of $f$ in $A(\beta)$. It follows from the construction of $\widehat{f}_{t}$ that we may follow continuously the critical points with respect to $t$ (cf. Prop. 3.1 in [10]). We may assume that $c_{1}$ is on the boundary of a petal of $\beta_{f}$ defined as a preimage of a right half-plane and that $\Phi_{f}\left(c_{1}\right)=0$.

The perturbation $\left(\widehat{f}_{t}\right)_{t}$ enables us to follow continuously each critical point of $C(f) \cap A\left(\beta_{f}\right)$ (cf. Prop. 3.1 in [10]). This correspondence extends to $\left(\widehat{F}_{t}\right)_{t}$. Hence we obtain a correspondence between $C(f) \cap A\left(\beta_{f}\right)$ and $C(F) \cap$ $A\left(\beta_{F}\right)$ (cf. Prop. 3.1). We denote by $H\left(c_{j}\right)$ the point corresponding to $c_{j}$. We normalize $\Phi_{F}$ so that $\Phi_{F}\left(H\left(c_{1}\right)\right)=0$.

We let $\left\{\widehat{\gamma}_{j}: 1 \leq j \leq \nu\right\}$ be pairwise disjoint curves of the plane invariant under the map $T: z \mapsto z+1$ such that $\Phi_{F}\left(c_{j}\right) \in \widehat{\gamma}_{j}$ for all $j$. These curves define an order on the critical points since each splits the plane in two components. Let also $\widehat{\gamma}_{j}^{+}$be the closure of the forward $T$-invariant component of $\widehat{\gamma}_{j} \backslash\left\{\Phi_{f}\left(c_{j}\right)\right\}$ (so it contains $\Phi_{f}\left(c_{j}\right)$ ), and $\gamma_{j}$ be the connected component of $\Phi_{f}^{-1}\left(\widehat{\gamma}_{j}^{+}\right)$which contains $c_{j}$. This curve joins $c_{j}$ to finitely many preimages of $\beta_{f}$. The perturbation which defines $\left(\widehat{f}_{t}\right)_{t}$ enables us also to follow continuously $\gamma_{j}$ as a curve joining the critical point to preimages of the new attracting points (cf. Prop. 2.1 of [10]). Applying $\phi$ defines curves which join $H\left(c_{j}\right)$ to preimages of $\beta_{F}$ which are the images under $H$ of the corresponding preimages of $\beta_{f}$. We note that we may choose the pinching deformation $\left(F_{t}, \phi_{t}\right)$ so that the yellow set $\mathcal{Y}$ be disjoint of these curves. The Fatou coordinate $\Phi_{F}$ defines an order on $H\left(c_{j}\right)$ which is the same as the previous one. This means that we may define curves $\delta_{j}:[0,1] \rightarrow \mathbb{C}$ joining $\Phi_{f}\left(c_{j}\right)$ to $\Phi_{F}\left(c_{j}\right)$ such that their quotients $\pi\left(\delta_{j}\right)$ in $\mathbb{C} / \mathbb{Z}$ are pairwise disjoint, where $\pi: \mathbb{C} \rightarrow \mathbb{C} / \mathbb{Z}$ is the canonical projection. We let $D_{j} \subset \mathbb{C} / \mathbb{Z}$ be pairwise disjoint neighborhoods of $\pi\left(\delta_{j}\right)$. We choose them so that these domains are disjoint from $\pi \circ \Phi_{F} \circ \phi(\mathcal{Y})$. Therefore, there is a quasiconformal isotopy $\left(\bar{\omega}_{s}\right)_{0 \leq s \leq 1}$ defined on $\mathbb{C} / \mathbb{Z}$, supported on $\bigcup_{j} D_{j}$, such that $\bar{\omega}_{0}=\mathrm{id}$ and $\bar{\omega}_{s}\left(\pi \Phi_{f}\left(c_{j}\right)\right)=\pi \delta_{j}(s)$. Hence, there is a lift $\left(\omega_{s}\right)_{s}$ of $\left(\bar{\omega}_{s}\right)_{s}$ to $\mathbb{C}$ such that $\omega_{0}=$ id and $\omega_{1}\left(\Phi_{f}\left(c_{j}\right)\right)=\Phi_{F}\left(H\left(c_{j}\right)\right)$.

We claim that there is a map $\widetilde{\omega}: A\left(\beta_{f}\right) \rightarrow A\left(\beta_{F}\right)$ such that $\Phi_{F} \circ \widetilde{\omega}=$ $\omega_{1} \circ \Phi_{f}$ and $\widetilde{\omega}\left(c_{j}\right)=H\left(c_{j}\right)$. This follows from the facts that the critical points of Fatou coordinates are the precritical points of the polynomials and that their critical points are associated to the corresponding preimages of the parabolic points. Furthermore, $\widetilde{\omega}$ maps petals to petals, so $\widetilde{\omega}\left(\beta_{f}\right)=\beta_{F}$, and the extension to the boundary coincides with $H$. We denote by $H$ this homeomorphism. Another application of Theorem 2.12 implies that $H$ is a global quasiconformal map which conjugates $f$ to $F$.

SteP 3. We first define an $F_{0}$-invariant ellipse field $E$. On $\mathbb{C} \backslash \phi(\mathcal{Y})$, we let $E=(H \circ \phi)^{*} \mathbb{S}^{1}$, and on $\phi(\mathcal{Y})$, we let $E=\mathbb{S}^{1}$ be the field of circles representing the standard complex structure. Let $\chi$ be given by the measurable Riemann 
mapping theorem with $\chi(z)=z+o(1)$ at infinity so that $f_{0}=\chi \circ F_{0} \circ \chi^{-1}$ is also monic and centered. Since $\chi$ is conformal on $\mathcal{Y}$, we may transport the simple pinching deformation defined for $F_{0}$ to $f_{0}$. We let $\left(f_{t}, \varphi_{t}\right)$ be this new deformation, and $\left(f_{1}, \varphi\right)$ be the limit of this deformation provided by Theorem 2.1. We note that since the fibers of $\phi$ and of $\varphi \circ \chi$ are the same, we may define a homeomorphism $\varphi \circ \chi \circ(H \circ \phi)^{-1}$ of the sphere which is conformal off $J_{F}$ and which conjugates $f$ to $f_{1}$. It follows from Theorem 2.12 that this homeomorphism is an affine map which has to be the identity thanks to the normalization.

We assume the reader is familiar with Thurston obstructions, Levy cycles etc. as in [24].

Proof of Proposition 3.8. Suppose that $(f, g, q, R)$ is a geometric mating of geometrically finite polynomials. We wish to prove that $\mathcal{T}(f)$ and $\mathcal{T}(g)$ are matable.

It follows from Proposition 3.6 that there is a homeomorphism $h_{f}$ : $J_{\mathcal{T}(f)} \rightarrow J_{f}$ which conjugates $\mathcal{T}(f)$ to $f$. We let $H_{f}: \mathbb{C} \rightarrow \mathbb{C}$ be a homeomorphic extension of $h_{f}$ which is conformal off $K_{\mathcal{T}(f)}$ (cf. the Remark following the statement of Prop. 3.6 or Cor. 3.10). We define similarly $h_{g}$ and $H_{g}$ for $g$.

The map

$$
H=q \circ\left(H_{f} \Perp H_{g}\right): K_{\mathcal{T}(f)} \sqcup K_{\mathcal{T}(g)} / \sim_{\text {ray }} \rightarrow \overline{\mathbb{C}}
$$

is a homeomorphism which maps $J_{\mathcal{T}(f)} \sqcup J_{\mathcal{T}(g)} / \sim_{\text {ray }}$ onto $J_{R}$. Define $T=$ $H \circ(\mathcal{T}(f) \Perp \mathcal{T}(g)) \circ H^{-1}$ on the Riemann sphere. It follows that $T$ is a postcritically finite ramified covering which agrees with $R$ on $J_{R}$.

Claim 1. T has no Thurston obstruction.

Assume that $T$ has an obstruction $\Gamma$. For each parabolic point of $R$ and in each component of its immediate basin, we consider a hyperbolic geodesic ray which joins the point in $\operatorname{Post}(T)$ to the parabolic point. The union of these curves produces a finite set of star-like graphs $\mathcal{S}$ as a simple pinching combinatorics. Let us remark that, for each parabolic point, we can include the critical orbits of these immediate basins (and only those) into a Jordan domain which can be contracted onto the star-like graph attached to this parabolic point. Let $\Omega$ be the union of these domains.

If $\Gamma$ can be homotoped rel. Post $(T)$ so that it does not intersect $\mathcal{S}$, then we may assume that $\Gamma$ is disjoint from $\Omega$. Therefore, $\Gamma$ is also an obstruction for $R$, which is impossible by Theorem B.4 in [16]. Hence $\Gamma$ intersects $\mathcal{S}$. By the intersection theory of [24], this in turn implies that $\Gamma$ is a Levy cycle. Therefore, there is a curve $\gamma$ cutting $\mathcal{S}$ and a preimage $\gamma^{\prime}$ by some iterate $(\mathcal{T}(f) \Perp \mathcal{T}(g))^{k}$ isotopic to $\gamma$ rel. the postcritical set such that $(\mathcal{T}(f) \Perp \mathcal{T}(g))^{k}: \gamma^{\prime} \rightarrow \gamma$ is a homeomorphism. 
The application of Proposition 3.11 produces simple pinchings $\left(F_{t}, \varphi_{t}\right)$ and $\left(G_{t}, \psi_{t}\right)$ of $F=F_{0}=\mathcal{S}(f)$ and $G=G_{0}=\mathcal{S}(g)$ which converge to $F_{1}=f$ and $G_{1}=g$. Let us consider maps $h_{F}, h_{G}, H_{F}, H_{G}$ and

$$
H_{0}=q \circ\left(H_{F} \Perp H_{F}\right): K_{F} \sqcup K_{G} / \sim_{\text {ray }} \rightarrow \overline{\mathbb{C}}
$$

as above. Let also $S=H_{0} \circ(F \Perp G) \circ H_{0}^{-1}$.

It follows that $\Gamma$ is also a Levy cycle for $S$ which intersects the pushforward $L$ of the simple pinching combinatorics.

By pushing $\gamma$ and $\gamma^{\prime}$ with $q \circ\left(\varphi_{1} \Perp \psi_{1}\right) \circ H_{0}^{-1}$, one can extract curves $\ell$ and $\ell^{\prime}$ in $\overline{\mathbb{C}}$ such that:

- $\ell$ and $\ell^{\prime}$ join parabolic points of $R$ in their repelling directions, or join a parabolic point in its repelling direction to a repelling postcritical point,

- $\ell^{\prime}$ is a preimage of $\ell$ by an iterate $R^{k^{\prime}}$,

- $\ell^{\prime}$ is isotopic to $\ell$ rel. the postcritical set of $R$,

- all curves in their isotopy class have definite diameter, and

$-R^{k^{\prime}}: \ell^{\prime} \rightarrow \ell$ is a homeomorphism.

This yields a contradiction because of Fatou's shrinking lemma (this is to be compared to the notion of degenerate combinatorial equivalence of Cui in [4], and was inspired by the proof of Theorem A therein). This proves the claim.

Since $T$ has no obstruction, Thurston's characterization of rational maps implies that $T$ is combinatorially equivalent to a rational map $R_{T}$. It follows from Theorem 2.1 in [22] and its proof that there is a continuous map $h: \overline{\mathbb{C}} \rightarrow \overline{\mathbb{C}}$, obtained as a uniform limit of homeomorphisms, such that $h \circ T=R_{T} \circ h$ and its restriction

is a homeomorphism.

$$
\left.h\right|_{\overline{\mathbb{C}} \backslash J_{R}}: \overline{\mathbb{C}} \backslash J_{R} \rightarrow \overline{\mathbb{C}} \backslash J_{R_{T}}
$$

Claim 2. $h$ is a homeomorphism of $\overline{\mathbb{C}}$.

It remains to prove that $h$ is injective on $J_{R}$. Recall that $h \circ R=R_{T} \circ h$ on $J_{R}$.

Since $h$ is a limit of homeomorphisms, it follows that preimages of connected sets are connected and full. Furthermore, since $R$ and $R_{T}$ are both degree $d$ mappings, if $K^{\prime}$ is a connected component of $R_{T}^{-n}(K)$ for some continuum $K \subset J_{R}$ and the degree of $\left.R_{T}\right|_{K^{\prime}}$ is $\delta$, then the restriction of $R$ to $h^{-1}\left(K^{\prime}\right)$ is also a degree $\delta$ mapping onto $h^{-1}(K)$.

We work with the spherical metric.

Since $R_{T}$ is subhyperbolic, a maximal degree $\delta$ and a radius $r>0$ exist such that, for any $z \in J_{R_{T}}$, the degree of the restriction of $R_{T}^{n}$ to any connected component of $R_{T}^{-n}(D(z, r))$ is bounded by $\delta$. 
Since $R$ is geometrically finite, a weaker statement remains true: there is an $r^{\prime}>0$ such that, for any $\varepsilon>0$ and any continuum $K \subset J_{R}$ of diameter less than $r^{\prime}$, there is an iterate $n_{0}$ such that for any $n \geq n_{0}$, any connected component $L$ of $R^{-n}(K)$ has diameter at most $\varepsilon$ (see for instance Prop. 3.1 in [11]).

We note that the diameter of $h^{-1}(\{z\})$ is bounded uniformly in $z$, so, using the (uniform) local connectivity of $J_{R}$, there is some integer $N$ independent of $z$ such that we may cover $h^{-1}(\{z\})$ in $J_{R}$ by at most $N$ continua of diameter less than $r^{\prime}$. Fix $z \in J_{R_{T}}$ and set $K_{n}=h^{-1}\left(R_{T}^{n}(z)\right)$. For $\varepsilon>0$, let $n$ be such that the diameter of any connected component of $R^{-n}(K)$ of any continuum $K \subset J_{R}$ of diameter less than $r^{\prime}$ is less than $\varepsilon /(\delta N)$. Cover $K_{n}$ by $N$ continua of diameter at most $r^{\prime}$. It follows that $h^{-1}(\{z\})$ is covered by at most $N \delta$ continua of diameter at most $\varepsilon /(N \delta)$, so that the diameter of $h^{-1}(\{z\})$ is less than $\varepsilon$. This implies that $h^{-1}(\{z\})$ is a point, and it establishes the claim that $h$ is a homeomorphism.

Therefore, $\left(\mathcal{T}(f), \mathcal{T}(g), h \circ H, R_{T}\right)$ defines a geometric marked mating, and this establishes Theorem D.

We may now prove Theorem E, which we recall in a more precise form:

COROLlary 3.12. If $(f, g, q, R)$ is a geometrically finite marked mating with at least one parabolic point, then there exist subhyperbolic perturbations $\left(f_{t}\right)_{t \in[0,1)}$ and $\left(g_{t}\right)_{t \in[0,1)}$ which converge to $f$ and $g$ respectively as $t$ tends to 1 such that $J_{f_{t}} \approx J_{f}, J_{g_{t}} \approx J_{g}$, and their matings $\left(f_{t}, g_{t}, q_{t}, R_{t}\right)$ exist and converge to $(f, g, q, R)$.

Proof. Applying Proposition 3.11 to $f$ and $g$ we obtain two pinching deformations $\left(f_{t}, \varphi_{t}\right)_{t \in[0,1)}$ and $\left(g_{t}, \psi_{t}\right)_{t \in[0,1)}$. By Theorem $\mathrm{D}$, there are $q_{0}, R_{0}$ so that $\left(f_{0}, g_{0}, q_{0}, R_{0}\right)$ is a marked mating. Now Theorem $\mathrm{B}$ ensures that the path of marked matings $\left(f_{t}, g_{t}, q_{t}, R_{t}\right)$ exists and converges to a marked mating $\left(f, g, q_{1}, R_{1}\right)$. Now $R_{1}$ is again geometrically finite and has parabolic points, so it is not a Lattès example. We can then apply the unicity result of Proposition 3.1 to conclude that $R=H R_{1} H^{-1}$ with $H$ a Möbius transformation. If $\widehat{q}_{t}=H \circ q_{t}$ and $\widehat{R}_{t}=H R_{t} H^{-1}$, then $\left(f_{t}, g_{t}, \widehat{q}_{t}, \widehat{R}_{t}\right)_{t}$ is again a path of marked matings and it converges to $\left(f, g, H \circ q_{1}, R\right)$. Now $H \circ q_{1}$ and $q$ differ by a Möbius map $G$ with $G R=R G$. We may then re-adjust the mating path as above to make it converge to $(f, g, q, R)$.

\section{A. QUOTIENT TOPOLOGY}

Definition. Let $A, B$ be two compact Hausdorff topological spaces and $F: A \rightarrow B$ be a continuous surjective map. We define the equivalence relation $\sim_{F}$ on $A$ by: $x \sim_{F} y$ if and only if $F(x)=F(y)$. 
We give several topological lemmas, admitting the first two.

Lemma A.1. The equivalence relation $\sim_{F}$ is closed (i.e. if $x_{n} \sim_{F} y_{n}$, $x_{n} \rightarrow x, y_{n} \rightarrow y$ then $\left.x \sim_{F} y\right)$, and $[F]:[x]_{F} \rightarrow F(x)$ is a homeomorphism from $A / \sim_{F}$ to $B$.

Lemma A.2. Let $\left(X_{i}, \sim_{i}\right), i=1,2$, be two topological spaces equipped with an equivalence relation each. Let $h: X_{1} \rightarrow X_{2}$ be continuous such that if $x \sim_{1} x^{\prime}$ then $h(x) \sim_{2} h\left(x^{\prime}\right)$. Let $\pi_{i}: X_{i} \rightarrow X_{i} / \sim_{i}$ be the quotient projections. Then the quotient map $[h]: X_{1} / \sim_{1} \rightarrow X_{2} / \sim_{2}$ is well defined and continuous, and is surjective if $h$ is.

Lemma A.3. Let $A, B, C, D$ be compact Hausdorff topological spaces, and let $l: A \rightarrow C, r: A \rightarrow D$ and $s: C \rightarrow B$ be continuous surjective maps. In particular $s \circ l$ generates an equivalence relation in A. Assume that $x \sim_{r} x^{\prime} \Rightarrow l(x) \sim_{s} l\left(x^{\prime}\right)$. Then there is a continuous surjective map $v: D \rightarrow B$ such that $v \circ r=s \circ l$ and $\sim_{v}=r_{*}\left(\sim_{s o l}\right)\left(\right.$ where $r_{*}(\sim)$ denotes the pushed forward equivalence relation, that is, $x \sim_{r_{*}(\sim)} x^{\prime}$ if and only if $r^{-1}(x) \cup r^{-1}\left(x^{\prime}\right)$ belongs to a single equivalence class of $\left.\sim\right)$.

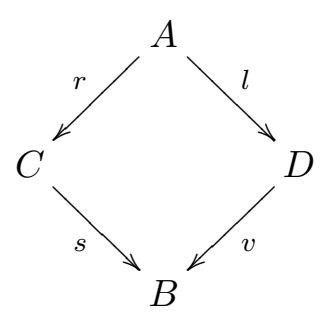

Proof. By Lemma A.1, $s$ and $r$ can be considered as quotient maps. The map $l$ satisfies the condition on $h$ in Lemma A.2. Therefore $v$ is well defined and continuous by Lemma A.2 with $v \circ r=s \circ l$. Since $s \circ l$ is surjective, $v$ is surjective.

Let $x, x^{\prime} \in D$. Now $r^{-1}(x) \sim_{s o l} r^{-1}\left(x^{\prime}\right)$ if and only if $s \circ l\left(r^{-1}(x)\right)=$ $s \circ l\left(r^{-1}\left(x^{\prime}\right)\right)$ if and only if $v(x)=v\left(x^{\prime}\right)$.

Lemma A.4. Let $g: \mathbb{S}^{2} \rightarrow \mathbb{S}^{2}$ be a continuous surjective map. For $t \in$ $[0,1)$, let $F_{t}, G_{t}: \mathbb{S}^{2} \rightarrow \mathbb{S}^{2}$ be two families of homeomorphisms of $\mathbb{S}^{2}$. Assume that, as $t \rightarrow 1, F_{t}$ and $G_{t}$ converge uniformly to continuous maps $F_{1}, G_{1}$ respectively, and $g$ maps each fiber of $F_{1}$ into a fiber of $G_{1}$. Then $g_{t}=$ $G_{t} \circ g \circ\left(F_{t}\right)^{-1}: \mathbb{S}^{2} \rightarrow \mathbb{S}^{2}$ converges uniformly to a continuous map $g_{1}$, and $g_{1} \circ F_{1}=G_{1} \circ g$.

Proof. Define $g_{1}=G_{1} \circ g \circ\left(F_{1}\right)^{-1}$.

1. $g_{1}$ is well defined and continuous. For this we apply Lemma A.3. Let $l=g, r=F_{1}$ and $s=G_{1}$. We can check that all the conditions of Lemma 
A.3 are satisfied and therefore $g_{1}=v$ is well defined, continuous, surjective and with fibers $F_{1 *}\left(\sim_{G_{1} \circ g}\right)$.

2. $g_{t}$ converges uniformly to $g_{1}$. In other words, for any $\varepsilon>0$, there is $t_{0}$ such that for any $1>t>t_{0}$ and any $y,\left|g_{t}(y)-g_{1}(y)\right|<\varepsilon$.

Assume by contradiction that $g_{t}$ does not converge uniformly to $g_{1}$. That is, there are $\varepsilon_{0}>0, t_{n} \rightarrow 1$ and $y_{n} \in \mathbb{S}^{2}$ such that $\left|g_{t_{n}}\left(y_{n}\right)-g_{1}\left(y_{n}\right)\right|>2 \varepsilon_{0}$.

We may assume $y_{n} \rightarrow y$ (by taking subsequences). Since $g_{1}$ is continuous, we have $g_{1}\left(y_{n}\right) \rightarrow g_{1}(y)$ and $\left|g_{t_{n}}\left(y_{n}\right)-g_{1}(y)\right|>\varepsilon_{0}$.

Let $x_{n}=\left(F_{t_{n}}\right)^{-1}\left(y_{n}\right)$. We may assume $x_{n} \rightarrow x$ (by taking subsequences). We claim that $F_{t_{n}}\left(x_{n}\right) \rightarrow F_{1}(x)$ and $g_{t_{n}} \circ F_{t_{n}}\left(x_{n}\right) \rightarrow g_{1} \circ F_{1}(x)$. The first limit is due to

$$
\left|F_{t_{n}}\left(x_{n}\right)-F_{1}(x)\right| \leq\left|F_{t_{n}}\left(x_{n}\right)-F_{1}\left(x_{n}\right)\right|+\left|F_{1}\left(x_{n}\right)-F_{1}(x)\right| \rightarrow 0 \text { as } n \rightarrow \infty
$$

by uniform convergence of $F_{t}$. The second limit can be proved similarly, by uniform convergence of $g_{t} \circ F_{t}$ to $g_{1} \circ F_{1}$, which is a consequence of the two equalities $g_{t} \circ F_{t}=G_{t} \circ g, G_{1} \circ g=g_{1} \circ F_{1}$ and the fact that $G_{t} \circ g$ converges uniformly to $G_{1} \circ g$.

Therefore $F_{1}(x)=y$ and $g_{t_{n}}\left(y_{n}\right)=g_{t_{n}} \circ F_{t_{n}}\left(x_{n}\right) \rightarrow g_{1} \circ F_{1}(x)=g_{1}(y)$. This leads to a contradiction.

3. $g_{1} \circ F_{1}=G_{1} \circ g$, since $g_{t} \circ F_{t}=G_{t} \circ g$ for $t \in[0,1)$ and, as $t \rightarrow 1$, all maps in the equation converge uniformly to the corresponding maps.

\section{B. AN INEQUALITY}

Lemma B.1. Let $z, w \in \mathbb{C} \backslash\{0\}$ be points in the plane such that $|z-w|$ $<|w|$, and let $\Gamma$ be the family of rectifiable curves which separate $\{0, \infty\}$ from $\{z, w\}$. Then

$$
|z-w|>|w| \exp \frac{-2 \pi}{\Lambda(\Gamma)} .
$$

Proof. Let

$$
h(\zeta)=\frac{\zeta-w}{\zeta+w}
$$

be the Möbius transformation which maps $(0, \infty, w)$ to $(-1,1,0)$. Since

$$
|z+w|=|2 w+(z-w)| \geq 2|w|-|z-w|>|w|
$$

and $\left|\frac{z-w}{z+w}\right|<1$, the annulus

$$
A=\left\{\zeta \in \mathbb{C}:\left|\frac{z-w}{z+w}\right|<|\zeta|<1\right\}
$$

is well defined and nondegenerate. Therefore,

$$
\frac{1}{2 \pi} \log \left|\frac{z+w}{z-w}\right|=\bmod A \leq \frac{1}{\Lambda(h(\Gamma))}=\frac{1}{\Lambda(\Gamma)} .
$$


Hence

$$
|z-w| \geq|z+w| \exp \frac{-2 \pi}{\Lambda(\Gamma)} \geq|w| \exp \frac{-2 \pi}{\Lambda(\Gamma)}
$$

Corollary B.2. Let $z_{t}, w_{t}, a, b$ be four distinct points with $z_{t}, w_{t}$ depending on a parameter $t$. Assume that $d\left(w_{t},\{a, b\}\right) \geq C>0$ for all $t$. If $d\left(z_{t}, w_{t}\right) \rightarrow 0$, then $\Lambda\left(\Gamma_{\left(z_{t}, w_{t}\right),(a, b)}\right) \rightarrow 0$. Consequently, if $\Lambda\left(\Gamma_{\left(z_{t}, w_{t}\right),(a, b)}\right) \geq$ $C^{\prime}>0$, then $d\left(z_{t}, w_{t}\right) \geq C^{\prime \prime}>0$.

\section{References}

[1] L. Ahlfors, Lectures on Quasiconformal Mappings, Van Nostrand, 1966.

[2] - Conformal Invariants, McGraw-Hill, 1973.

[3] L. Carleson and T. W. Gamelin, Complex Dynamics, Springer, 1993.

[4] G.-Z. Cui, Dynamics of rational maps, topology, deformation and bifurcation, preprint, May 2002 (early version: Geometrically finite rational maps with given combinatorics, 1997).

[5] G. David, Solutions de l'équation de Beltrami avec $\|\mu\|_{\infty}=1$, Ann. Acad. Sci. Fenn. Ser. A 1 Math. 13 (1988), 25-70.

[6] A. Douady, Descriptions of compact sets in $\mathbb{C}$, in: Topological Methods in Modern Mathematics (Stony Brook, NY, 1991), Publish or Perish, Houston, TX, 1993, 429465.

[7] A. Douady and J. H. Hubbard, On the dynamics of polynomial-like mappings, Ann. Sci. École Norm. Sup. (4) 18 (1985), 287-343.

[8] A. Epstein, Counterexamples to the quadratic mating conjecture, manuscript, 1997.

[9] P. Haïssinsky, Chirurgie parabolique, C. R. Acad. Sci. Paris Sér. I Math. 327 (1998), 195-198.

[10] —, Déformation J-équivalente de polynômes géométriquement finis, Fund. Math. 163 (2000), 131-141.

[11] —, Rigidity and expansion for rational maps, J. London Math. Soc. (2) 63 (2001), 128-140.

[12] —, Pincements de polynômes, Comment. Math. Helv. 77 (2002), 1-23.

[13] M. Lyubich and Y. Minsky, Laminations in holomorphic dynamics, J. Differential Geom. 47 (1997), 17-94.

[14] R. Mañé, On a theorem of Fatou, Bol. Soc. Brasil. Mat. 24 (1993), 1-11.

[15] C. T. McMullen, Automorphisms of rational maps, in: Holomorphic Functions and Moduli, Vol. I (Berkeley, CA, 1986), Math. Sci. Res. Inst. Publ., 10, Springer, New York, 1988, 31-60.

[16] —, Complex Dynamics and Renormalization, Ann. of Math. Stud. 135, Princeton Univ. Press, 1994.

[17] —, Hausdorff dimension and conformal dynamics II: Geometrically finite rational maps, Comment. Math. Helv. 75 (2000), 535-593.

[18] J. Milnor, Pasting together Julia sets, Exp. Math., to appear.

[19] —, Dynamics in One Complex Variable: Introductory Lectures, Vieweg, 1999.

[20] M. Rees, Realization of matings of polynomials as rational maps of degree two, manuscript, 1986.

[21] S. Rickman, Removability theorems for quasiconformal mappings, Ann. Acad. Sci. Fenn. Ser. AI 449 (1969). 
[22] M. Shishikura, On a theorem of Mary Rees for the matings of polynomials, in: The Mandelbrot Set, Theme and Variations, L. Tan (ed.), London Math. Soc. Lecture Note Ser. 274, Cambridge Univ. Press, 2000, 289-305.

[23] M. Shishikura and L. Tan, An alternative proof of Mañé's theorem on non-expanding Julia sets, ibid. 265-279.

[24] —, 一, A family of cubic rational maps and matings of cubic polynomials, Exper. Math. 9 (2000), 29-53.

[25] L. Tan, Matings of quadratic polynomials, Ergodic Theory Dynam. Systems 12 (1992), 589-620.

[26] -, On pinching deformations of rational maps, Ann. Sci. École Norm. Sup. (4) 35 (2002), 353-370.

[27] - Existence and deformations of semi-rational maps, following Cui G.-Z., in: Proc. Bodilfest, P. Hjort and C. L. Petersen (eds.), to appear.

\section{LATP/CMI}

Université de Provence

39, rue Frédéric Joliot-Curie

13453 Marseille Cedex 13, France

E-mail: phaissin@cmi.univ-mrs.fr
Unité CNRS-UMR 8088

Département de Mathématiques Université de Cergy-Pontoise 2, avenue Adolphe Chauvin 95302 Cergy-Pontoise Cedex, France E-mail: tanlei@math.u-cergy.fr

Received 8 December 2003; in revised form 10 March 2004 